\title{
REAL ANALYTICITy OF HAUSDORFF Dimension FOR HIGHER DIMENSIONAL GRAPH DIRECTED MARKOV SYSTEMS
}

\author{
MARIO ROY AND MARIUSZ URBAŃSKI
}

\begin{abstract}
In this paper we prove that the Hausdorff dimension function of the limit sets of strongly regular, countable, conformal graph directed Markov systems living in higher dimensional Euclidean spaces $\mathbb{R}^{d}, d \geq 3$, and with an underlying finitely irreducible incidence matrix is real-analytic.
\end{abstract}

\section{INTRODUCTION}

In this paper we deal with the problem of regularity of the Hausdorff dimension function of the limit sets of strongly regular, countable, conformal graph directed Markov systems living in higher dimensional Euclidean spaces $\mathbb{R}^{d}, d \geq 3$, and whose underlying incidence matrix is finitely irreducible. The foundations of the theory of countable, conformal graph directed Markov systems were laid in [4]. This theory extends that of countable, conformal iterated function systems developed in [6]. Extensive research in this field has been done before. As we are mainly interested in this article in regularity properties of the Hausdorff dimension function, we would like to bring to the attention of our readers article [7], in which we explored the continuity of the Hausdorff dimension in the general case $(d \geq 1)$, as well as papers [7] (sections 6 and 8), [1] and [3], where the problem of regularity of the Hausdorff dimension was treated in the complex plane (case $d=2$ ), under the assumption that the generators of the systems are holomorphic. In [3] the real-analyticity was established under weaker assumptions (to our knowledge). In the present paper we go beyond the plane by investigating the case $d \geq 3$. In this case, the conformal generators of the systems are not analytic (unless they are affine), but in view of Liouville's Theorem (see [2], for instance) they all take on the same canonical form. This enables us to parametrize the space $M_{d}$ of such maps by $q=2+\frac{1}{2} d(d+3)$ real parameters, and thereafter define natural notions of differentiability and real-analyticity on the space $\left(\mathbb{R}^{q}\right)^{I}$, where $I$ is a countable set. In our framework $I$ is the alphabet of the graph directed Markov systems. Our main result, Theorem 7.2, asserts that the Hausdorff dimension function is real-analytic when this function is restricted to an open subset $W$ of $\left(\mathbb{R}^{q}\right)^{I}$ corresponding to a family of strongly regular, conformal graph directed Markov systems with a common alphabet $I$, a common finitely irreducible incidence matrix and which satisfy the open set condition.

Date: August 31, 2006.

1991 Mathematics Subject Classification. Primary: 37C45; Secondary: 37D45, 37D35, 32H50.

Key words and phrases. Holomorphic dynamics, Hausdorff dimension, Meromorphic functions.

Research of the first author was supported by NSERC (Natural Sciences and Engineering Research Council of Canada). Research of the second author was supported in part by the NSF Grant DMS 0400481. 
The main idea of the proof is to ascribe canonically to each graph directed Markov system $\Phi$ its complexification $\hat{\Phi}$ acting on $\hat{\mathbb{C}}^{d}$. This complexification is no longer conformal (with respect to the Euclidean metric on $\hat{\mathbb{C}}^{d}$ ) but it permits us to define a holomorphic extension of the normalized derivatives of the generators of $\Phi$ (see (6.2)). With the help of this extension we define the family of Perron-Frobenius operators associated to the family of complexified systems. These operators constitute a holomorphic family (see Lemma 6.4). This fact and the quasi-compactness property of these operators allow us to apply KatoRellich perturbation theorem to conclude that the corresponding pressure function is realanalytic. It then follows from the implicit function theorem that the only zero of this pressure function, which we call Bowen's parameter, is real-analytic throughout $W$ (see Theorem 7.1). It is important to note that in order to come to this conclusion we do not require that the open set condition be fulfilled. Whenever this latter is satisfied, a variant of Bowen's formula (see [4]), which identifies the Hausdorff dimension of the limit set of a conformal graph directed Markov system as the zero of the pressure function, permits us to conclude that the Hausdorff dimension function coincides with Bowen's parameter and thus is real-analytic (see Theorem 7.2). It is also worth pointing out that this fact was unknown until now even when the underlying alphabet $I$ of the systems is finite.

\section{Differentiability}

Throughout this section $\mathcal{F}$ denotes either $\mathbb{R}$ or $\mathbb{C}$. Let also $d \geq 1$ be an integer and $I$ a countable (either finite or infinite) set. For every $F \subset I$ we denote by $P_{F}:\left(\mathcal{F}^{d}\right)^{I} \rightarrow\left(\mathcal{F}^{d}\right)^{F}$ the canonical projection from $\left(\mathcal{F}^{d}\right)^{I}$ onto $\left(\mathcal{F}^{d}\right)^{F}$. For every point $x \in\left(\mathcal{F}^{d}\right)^{I}$ and every set $F \subset I$ set

$$
\mathcal{F}_{(x, F)}=P_{I \backslash F}^{-1}\left(P_{I \backslash F}(x)\right) .
$$

If the set $F$ is finite, then $\mathcal{F}_{(x, F)}$ is canonically isomorphic to $\mathcal{F}^{d(\# F)}$ and is called the space box of $\left(\mathcal{F}^{d}\right)^{I}$ generated by $x$ and $F$. Recall that the box topology on $\left(\mathcal{F}^{d}\right)^{I}$ has for a base the set of all Cartesian products $\prod_{i \in I} U_{i}$, where each $U_{i}, i \in I$, is an open subset of $\mathcal{F}^{d}$. A function $G: U \rightarrow B$ from $U$, an open subset of $\left(\mathcal{F}^{d}\right)^{I}$ in the box topology, to a Banach space $B$ is said to be differentiable at a point $x \in U$ if its restriction to every space box $\mathcal{F}_{(x, F)}$ intersected with $U$, that is $\left.G\right|_{\mathcal{F}_{(x, F)} \cap U}$, is differentiable at the point $x$. As usual, $G$ is said to be differentiable on a set $V \subset U$ if it is differentiable at every point of $V$. Analogous definitions of $C^{r}$ differentiability $(1 \leq r \leq \infty)$, real analyticity, and complex analyticity (that is, holomorphicity) when $\mathcal{F}=\mathbb{C}$, can be readily made. Note that due to Hartogs Theorem, it suffices to consider singleton sets $F$ in the definition of complex analyticity.

\section{Conformal Mappings}

We now turn our attention to conformal mappings in higher-dimensional spaces. Let us fix an integer $d \geq 3$. For every $z=\left(z_{1}, z_{2}, \ldots, z_{d}\right) \in \mathbb{C}^{d}$ we define

$$
\left\|z|\||^{2}:=\sum_{j=1}^{d} z_{j}^{2} \text { and }\right\| z \|^{2}:=\sum_{j=1}^{d}\left|z_{j}\right|^{2} .
$$

Of course, in general $\||z|\|^{2}$ is a complex number and is not the square of a norm. However, $\left\|\left.\left.|\cdot|\right|^{2}\right|_{\mathbb{R}^{d}}=\right\| \cdot \|\left.^{2}\right|_{\mathbb{R}^{d}}$ and $\|\cdot\|^{2}$ are the squares of the standard Euclidean norms in $\mathbb{R}^{d}$ 
and $\mathbb{C}^{d}$, respectively. It is also worth noticing that $\left|\left\||| z\left|\left\|^{2} \mid \leq\right\| z \|^{2}\right.\right.\right.$ for every $z \in \mathbb{C}^{d}$. We will often implicitly consider $\mathbb{R}^{d}$ as naturally embedded in $\mathbb{C}^{d}$. Open balls in $\mathbb{R}^{d}$ will be denoted by $B(z, r)$, while open balls in $\mathbb{C}^{d}$ will be denoted by $B_{\mathbb{C}^{d}}(z, r)$. We denote by $\hat{\mathbb{R}}^{d}$ and $\hat{\mathbb{C}}^{d}$ the one-point (Alexandrov) compactifications of $\mathbb{R}^{d}$ and $\mathbb{C}^{d}$, respectively, obtained by adding the point $\infty$. Recall that, given $a \in \mathbb{R}^{d}$ and $r>0$, the inversion $i_{a, r}: \hat{\mathbb{R}}^{d} \rightarrow \hat{\mathbb{R}}^{d}$ in the sphere centred at $a$ and of radius $r$ is given by the formula

$$
i_{a, r}(x)=r^{2} \frac{x-a}{\|x-a\|^{2}}+a .
$$

We further set $i_{\infty, \infty}=I d$. Obviously, $i_{a, r}$ is an involution (that is, $i_{a, r}^{2}=I d$ ) which leaves the sphere $\partial B(a, r)$ invariant, and $a=i_{a, r}^{-1}(\infty)$. Liouville's Theorem (see $[2,10]$ ) says that each $C^{1}$ conformal homeomorphism $\varphi$ defined on a connected, open subset of $\mathbb{R}^{d}$ extends to the entire space $\hat{\mathbb{R}}^{d}$ and takes on the form

$$
\varphi(x)=\lambda A \circ i_{a, r}(x)+b,
$$

where $\lambda>0, A: \mathbb{R}^{d} \rightarrow \mathbb{R}^{d}$ is a (linear) isometry, $a \in \hat{\mathbb{R}}^{d}, r \in(0, \infty]$ and $b \in \mathbb{R}^{d}$. The number $\lambda$ is often called the scalar factor of $\varphi$, and $a=\varphi^{-1}(\infty)$ its center of inversion. Note that for every $x \in \mathbb{R}^{d}$, the scaling factor of the derivative $\varphi^{\prime}(x)$ is given by the formula

$$
\left\|\varphi^{\prime}(x)\right\|=\frac{\lambda r^{2}}{\|x-a\|^{2}}>0
$$

For every $a \in \mathbb{R}^{d}$, let

$$
Z_{a}=\left\{z \in \mathbb{C}^{d}:\|\| z-a \|\left.\right|^{2}=0\right\} .
$$

Clearly, $Z_{a}=a+Z_{0}$. Notice that the inversion $i_{a, r}$ extends to the open set $\hat{\mathbb{C}}^{d} \backslash Z_{a}$. Indeed, for every $z \in \hat{\mathbb{C}}^{d} \backslash Z_{a}$, set

$$
i_{a, r}(z)=r^{2} \frac{z-a}{\|z-a\|^{2}}+a
$$

The $C^{1}$ conformal homeomorphism $\varphi: \hat{\mathbb{R}}^{d} \rightarrow \hat{\mathbb{R}}^{d}$ therefore extends canonically to a map from $\hat{\mathbb{C}}^{d} \backslash Z_{a}$ to $\hat{\mathbb{C}}^{d}$ by the formula

$$
\varphi(z)=\lambda A \circ i_{a, r}(z)+b .
$$

The "scaling factor of the derivative" of this extension is defined as

$$
\left\|\varphi^{\prime}(z)\right\| \|=\frac{\lambda r^{2}}{\|z-a\|^{2}}
$$

Observe that this factor is generally a nonzero complex number.

We will later need the following result:

Lemma 3.1. If $X$ is a compact subset of $\mathbb{R}^{d}$ and $V \supset X$ is an open subset of $\mathbb{R}^{d}$, then

$$
\operatorname{dist}_{\mathbb{C}^{d}}\left(X, \bigcup_{a \in \mathbb{R}^{d} \backslash V} Z_{a}\right)>0 .
$$


Proof. Suppose on the contrary that $\operatorname{dist}_{\mathbb{C}^{d}}\left(X, \cup_{a \in \mathbb{R}^{d} \backslash V} Z_{a}\right)=0$. Then there exist sequences $\left(a^{(n)}\right)_{n=1}^{\infty} \subset \mathbb{R}^{d} \backslash V$ and $\left(z^{(n)}\right)_{n=1}^{\infty} \subset \mathbb{C}^{d}$ such that $z^{(n)} \in Z_{a^{(n)}}$ for every $n \geq 1$ and $\lim _{n \rightarrow \infty} \operatorname{dist}_{\mathbb{C}^{d}}\left(X, z^{(n)}\right)=0$. Since $X$ is compact, by passing to a subsequence if necessary, we may assume without loss of generality that the sequence $\left(z^{(n)}\right)_{n=1}^{\infty}$ converges to a point $x \in X$. Now, for every $n \geq 1$, we have

$$
\begin{aligned}
\|\| z^{(n)}-a^{(n)} \|^{2} & =\sum_{j=1}^{d}\left(\left(z_{j}^{(n)}-x_{j}\right)+\left(x_{j}-a_{j}^{(n)}\right)\right)^{2} \\
& =\sum_{j=1}^{d}\left(z_{j}^{(n)}-x_{j}\right)^{2}+2 \sum_{j=1}^{d}\left(z_{j}^{(n)}-x_{j}\right)\left(x_{j}-a_{j}^{(n)}\right)+\sum_{j=1}^{d}\left(x_{j}-a_{j}^{(n)}\right)^{2} \\
& =\|\| z^{(n)}-x\|\|^{2}+2 \sum_{j=1}^{d}\left(z_{j}^{(n)}-x_{j}\right)\left(x_{j}-a_{j}^{(n)}\right)+\left\|x-a^{(n)}\right\|^{2} .
\end{aligned}
$$

To shorten the notation, set $\Delta:=\operatorname{dist}(X, \partial V)$. Since $a^{(n)} \in \mathbb{R}^{d} \backslash V$ and $x \in X$, we have

$$
\left\|x-a^{(n)}\right\|^{2} \geq \Delta^{2}>0 .
$$

Moreover, since $\lim _{n \rightarrow \infty} z^{(n)}=x$, there exists $N \geq 1$ such that for all $n \geq N$ we have

$$
\left\|z^{(n)}-x\right\| \leq \frac{1}{8 d} \min \left\{1, \Delta^{2}\right\} \text {. }
$$

So, for all $n \geq N$ and those $1 \leq j \leq d$ such that $\left|x_{j}-a_{j}^{(n)}\right| \leq 1$, we have

$$
2\left|z_{j}^{(n)}-x_{j}\right| \cdot\left|x_{j}-a_{j}^{(n)}\right| \leq 2\left|z_{j}^{(n)}-x_{j}\right| \leq 2\left\|z^{(n)}-x\right\| \leq \frac{1}{4 d} \Delta^{2},
$$

whereas for those $j$ for which $\left|x_{j}-a_{j}^{(n)}\right| \geq 1$, we get

$$
\begin{aligned}
2\left|z_{j}^{(n)}-x_{j}\right| \cdot\left|x_{j}-a_{j}^{(n)}\right| \leq 2\left|z_{j}^{(n)}-x_{j}\right| \cdot\left|x_{j}-a_{j}^{(n)}\right|^{2} & \leq 2\left\|z^{(n)}-x\right\| \cdot\left|x_{j}-a_{j}^{(n)}\right|^{2} \\
& \leq \frac{1}{4 d}\left|x_{j}-a_{j}^{(n)}\right|^{2} \leq \frac{1}{4 d}\left\|x-a^{(n)}\right\|^{2} .
\end{aligned}
$$

Thus, using (3.8) - (3.12), we obtain for every $n \geq N$

$$
\begin{aligned}
\left|\left\|\left|z^{(n)}-a^{(n)} \|\right|^{2} \mid\right.\right. & \geq\left\|x-a^{(n)}\right\|^{2}-\left\|z^{(n)}-x\right\|^{2}-2 \sum_{j=1}^{d}\left|z_{j}^{(n)}-x_{j}\right| \cdot\left|x_{j}-a_{j}^{(n)}\right| \\
& \geq\left\|x-a^{(n)}\right\|^{2}-\frac{1}{4} \Delta^{2}-\frac{1}{4 d} \sum_{j=1}^{d} \max \left\{\Delta^{2},\left\|x-a^{(n)}\right\|^{2}\right\} \\
& \geq\left\|x-a^{(n)}\right\|^{2}-\frac{1}{4}\left\|x-a^{(n)}\right\|^{2}-\frac{1}{4}\left\|x-a^{(n)}\right\|^{2}=\frac{1}{2}\left\|x-a^{(n)}\right\|^{2} \\
& \geq \frac{1}{2} \Delta^{2}>0 .
\end{aligned}
$$

Hence $z^{(n)} \notin Z_{a^{(n)}}$ for each $n \geq N$, and this contradiction completes the proof. 
We will also need the following estimates:

Lemma 3.2. Let $X$ be a compact subset of $\mathbb{R}^{d}$ and $V \supset X$ an open subset of $\mathbb{R}^{d}$. Let $\Delta:=\operatorname{dist}(X, \partial V)>0$ and $\tilde{\Delta}=\Delta /(32 d)$. Then for all $(w, \alpha) \in B_{\mathbb{C}^{d}}(X, \tilde{\Delta}) \times B_{\mathbb{C}^{d}}\left(\mathbb{R}^{d} \backslash V, \tilde{\Delta}\right)$ we have

$$
|||| w-\alpha\left|\left\|^{2} \mid \geq \frac{3}{16}\right\| w-\alpha \|^{2}>0 .\right.
$$

Proof. Let $(w, \alpha) \in B_{\mathbb{C}^{d}}(X, \tilde{\Delta}) \times B_{\mathbb{C}^{d}}\left(\mathbb{R}^{d} \backslash V, \tilde{\Delta}\right)$ and take $(x, a) \in X \times\left(\mathbb{R}^{d} \backslash V\right)$ such that $(w, \alpha) \in B_{\mathbb{C}^{d}}(x, \tilde{\Delta}) \times B_{\mathbb{C}^{d}}(a, \tilde{\Delta})$. Then

$$
\begin{aligned}
& |||| w-\alpha||^{2}|=||| w-x+x-a+a-\alpha\left|\|^{2}\right| \\
& =\left|\begin{array}{l}
\|w-x\|\left\|^{2}+\right\| x-a\left\|^{2}+\right\||| a-\alpha\|\|^{2} \\
+2 \sum_{j=1}^{d}\left[\left(w_{j}-x_{j}\right)\left(x_{j}-a_{j}\right)+\left(x_{j}-a_{j}\right)\left(a_{j}-\alpha_{j}\right)+\left(w_{j}-x_{j}\right)\left(a_{j}-\alpha_{j}\right)\right]
\end{array}\right| \\
& \geq \quad\|x-a\|^{2}-|||| w-x||^{2}|-||||a-\alpha| \|^{2} \mid \\
& -2 \sum_{j=1}^{d}\left[\left|w_{j}-x_{j}\right| \cdot\left|x_{j}-a_{j}\right|+\left|x_{j}-a_{j}\right| \cdot\left|a_{j}-\alpha_{j}\right|+\left|w_{j}-x_{j}\right| \cdot\left|a_{j}-\alpha_{j}\right|\right] \\
& \geq\|x-a\|^{2}-\|w-x\|^{2}-\|a-\alpha\|^{2} \\
& -2 d[\|w-x\| \cdot\|x-a\|+\|x-a\| \cdot\|a-\alpha\|+\|w-x\| \cdot\|a-\alpha\|] \\
& \geq\|x-a\|^{2}-2 \tilde{\Delta}^{2}-2 d\left[2 \tilde{\Delta}\|x-a\|+\tilde{\Delta}^{2}\right] \\
& =\|x-a\|^{2}\left(1-\frac{4 \tilde{\Delta} d}{\|x-a\|}\right)-2(d+1) \tilde{\Delta}^{2} \\
& \geq\|x-a\|^{2}\left(1-\frac{4 \tilde{\Delta} d}{\Delta}\right)-2(d+1) \tilde{\Delta}^{2} \\
& =\|x-a\|^{2}\left(1-\frac{1}{8}\right)-\frac{2(d+1) \Delta^{2}}{1024 d^{2}} \\
& \geq \frac{7}{8}\|x-a\|^{2}-\frac{2(d+1)\|x-a\|^{2}}{1024 d^{2}} \\
& \geq \frac{3}{4}\|x-a\|^{2} \text {. }
\end{aligned}
$$

Since

$$
\begin{aligned}
\|x-a\| & \geq\|w-\alpha\|-\|x-w\|-\|\alpha-a\| \\
& \geq\|w-\alpha\|-2 \tilde{\Delta} \\
& \geq \frac{1}{2}\|w-\alpha\| \geq \frac{1}{2}(\Delta-2 \tilde{\Delta})>0
\end{aligned}
$$

we conclude that

$$
\left|\||w-\alpha|\|^{2}\right| \geq \frac{3}{4}\left(\frac{1}{2}\|w-\alpha\|\right)^{2}=\frac{3}{16}\|w-\alpha\|^{2} \geq \frac{3}{16}(\Delta-2 \tilde{\Delta})^{2}>0 .
$$


Lemma 3.3. If $X$ is a compact subset of $\mathbb{R}^{d}$ and $V \supset X$ is an open subset of $\mathbb{R}^{d}$, then there exists a constant $C(X, V)>0$, depending only on $\Delta:=\operatorname{dist}(X, \partial V)$ and $d$, such that for all $(z, \alpha),(w, \beta) \in B_{\mathbb{C}^{d}}(X, \tilde{\Delta}) \times B_{\mathbb{C}^{d}}\left(\mathbb{R}^{d} \backslash V, \tilde{\Delta}\right)$, where $\tilde{\Delta}:=\Delta /(32 d)$, we have

$$
\begin{aligned}
\max \left\{\left|\frac{\|z-\alpha\|^{2}}{\|w-\beta\|^{2}}-1\right|,\left|\frac{\|\mid z-\alpha\|^{2}}{\|w-\beta\|^{2}}-1\right|\right\} & \\
& \leq C(X, V)\left(\|z-w\|^{2}+\|\alpha-\beta\|^{2}+\|z-w\|+\|z-w\| \cdot\|\alpha-\beta\|+\|\alpha-\beta\|\right) .
\end{aligned}
$$

Proof. Let $(z, \alpha),(w, \beta) \in B_{\mathbb{C}^{d}}(X, \tilde{\Delta}) \times B_{\mathbb{C}^{d}}\left(\mathbb{R}^{d} \backslash V, \tilde{\Delta}\right)$. Then

$$
\begin{aligned}
& \left.\left|\frac{\|z-\alpha\|^{2}}{\|w-\beta\|^{2}}-1\right|=\mid \frac{\left\{\begin{array}{l}
\|z-w\|^{2}+\|w-\beta\|^{2}+\|\beta-\alpha\|^{2} \\
+2 \sum_{j=1}^{d} \operatorname{Re}\left[\left(z_{j}-w_{j}\right) \overline{\left(w_{j}-\beta_{j}\right)}+\left(z_{j}-w_{j}\right) \overline{\left(\beta_{j}-\alpha_{j}\right)}+\left(w_{j}-\beta_{j}\right) \overline{\left(\beta_{j}-\alpha_{j}\right)}\right]
\end{array}\right\}}{\|w-\beta\|^{2}}-1\right\} \\
& =\frac{\left|\begin{array}{l}
\|z-w\|^{2}+\|\alpha-\beta\|^{2} \\
+2 \sum_{j=1}^{d} \operatorname{Re}\left[\left(z_{j}-w_{j}\right) \overline{\left(w_{j}-\beta_{j}\right)}+\left(z_{j}-w_{j}\right) \overline{\left(\beta_{j}-\alpha_{j}\right)}+\left(w_{j}-\beta_{j}\right) \overline{\left(\beta_{j}-\alpha_{j}\right)}\right]
\end{array}\right|}{\|w-\beta\|^{2}} \\
& \leq \frac{\left\{\begin{array}{l}
\|z-w\|^{2}+\|\alpha-\beta\|^{2} \\
+2 \sum_{j=1}^{d}\left[\left|\left(z_{j}-w_{j}\right) \overline{\left(w_{j}-\beta_{j}\right)}\right|+\left|\left(z_{j}-w_{j}\right) \overline{\left(\beta_{j}-\alpha_{j}\right)}\right|+\left|\left(w_{j}-\beta_{j}\right) \overline{\left(\beta_{j}-\alpha_{j}\right)}\right|\right]
\end{array}\right\}}{\|w-\beta\|^{2}} \\
& \leq \frac{\left\{\begin{array}{l}
\|z-w\|^{2}+\|\alpha-\beta\|^{2} \\
+2 d[\|z-w\| \cdot\|w-\beta\|+\|z-w\| \cdot\|\beta-\alpha\|+\|w-\beta\| \cdot\|\beta-\alpha\|]
\end{array}\right.}{\|w-\beta\|^{2}} \\
& \leq \begin{aligned}
\left(\Delta^{\prime}\right)^{-2}(\| z & \left.-w\left\|^{2}+\right\| \alpha-\beta \|^{2}\right) \\
& +2 d\left(\Delta^{\prime}\right)^{-1}\left(\|z-w\|+\left(\Delta^{\prime}\right)^{-1}\|z-w\| \cdot\|\alpha-\beta\|+\|\alpha-\beta\|\right),
\end{aligned}
\end{aligned}
$$

where $\Delta^{\prime}=\Delta-2 \tilde{\Delta}$ 
Similarly,

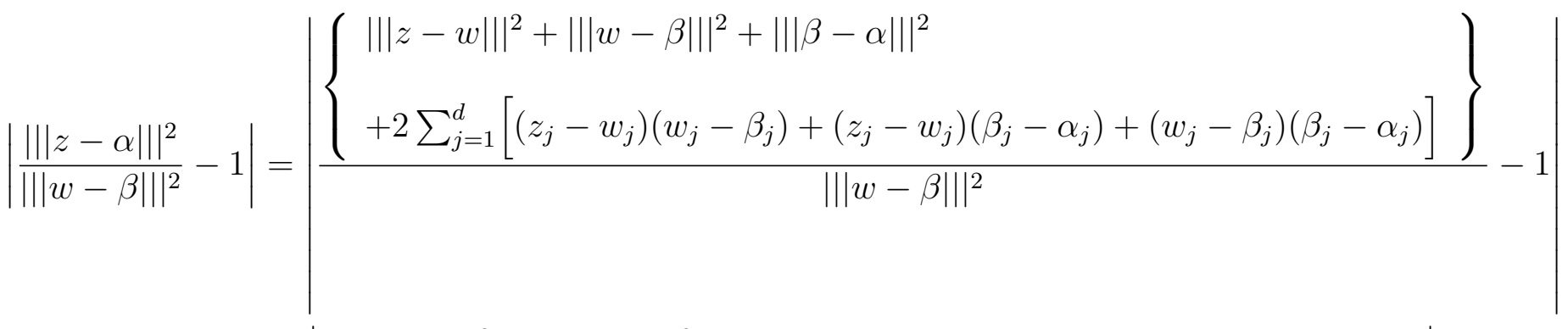

$$
\begin{aligned}
& =\frac{\left|\begin{array}{l}
\left\|z-w\left|\left\|^{2}+\right\| \alpha-\beta\right|\right\|^{2} \\
+2 \sum_{j=1}^{d}\left[\left(z_{j}-w_{j}\right)\left(w_{j}-\beta_{j}\right)+\left(z_{j}-w_{j}\right)\left(\beta_{j}-\alpha_{j}\right)+\left(w_{j}-\beta_{j}\right)\left(\beta_{j}-\alpha_{j}\right)\right]
\end{array}\right|}{|\|w-\beta\||^{2} \mid} \\
& \leq \frac{\left\{\begin{array}{l}
\|z-w\|^{2}+\|\alpha-\beta\|^{2} \\
+2 d[\|z-w\| \cdot\|w-\beta\|+\|z-w\| \cdot\|\beta-\alpha\|+\|w-\beta\| \cdot\|\beta-\alpha\|]
\end{array}\right\}}{(3 / 16)\|w-\beta\|^{2}} \\
& \leq \frac{16}{3}\left\{\begin{array}{r}
\left(\Delta^{\prime}\right)^{-2}\left(\|z-w\|^{2}+\|\alpha-\beta\|^{2}\right) \\
+2 d\left(\Delta^{\prime}\right)^{-1}\left(\|z-w\|+\left(\Delta^{\prime}\right)^{-1}\|z-w\| \cdot\|\alpha-\beta\|+\|\alpha-\beta\|\right)
\end{array}\right\}
\end{aligned}
$$

where we have relied upon Lemma 3.2 to bound from below the denominator in the secondto-last inequality. Simply take $C(X, V)=(32 / 3) \max \left\{\left(\Delta^{\prime}\right)^{-2}, 6 d\left(\Delta^{\prime}\right)^{-1} \max \left\{1,\left(\Delta^{\prime}\right)^{-1}\right\}\right\}$.

We will also need a slightly different type of estimates:

Lemma 3.4. Let $X$ be a compact subset of $\mathbb{R}^{d}$ and $V \supset X$ an open subset of $\mathbb{R}^{d}$. Let $\Delta:=\operatorname{dist}(X, \partial V)>0$ and $\tilde{\Delta}:=\Delta /(32 d)$. Then for every $0<r<\tilde{\Delta}$ and every couple $(z, \alpha) \in B_{\mathbb{C}^{d}}(X, r) \times B_{\mathbb{C}^{d}}\left(\mathbb{R}^{d} \backslash V, r\right)$ we have

$$
\left|\frac{\|z-\alpha\|^{2}}{\|\| z-\alpha \|^{2}}-1\right| \leq \frac{64 d r}{3\|z-\alpha\|} .
$$

Proof. Let $(z, \alpha) \in B_{\mathbb{C}^{d}}(X, r) \times B_{\mathbb{C}^{d}}\left(\mathbb{R}^{d} \backslash V, r\right)$. Then 


$$
\begin{aligned}
& \left|\frac{\|z-\alpha\|^{2}}{\|\| z-\alpha \|^{2}}-1\right|=\left|\frac{\|z-\alpha\|^{2}-|\|z-\alpha\||^{2}}{\||| z-\alpha||^{2}}\right| \\
& =\frac{\left|\|z-\alpha\|^{2}-\left\||| z-\alpha\left|\|^{2}\right|\right.\right.}{\left|\left\|z-\alpha\left|\|^{2}\right|\right.\right.} \\
& \leq \frac{16}{3} \frac{\left|\sum_{j=1}^{d}\left(z_{j}-\alpha_{j}\right)\left(\overline{z_{j}}-\overline{\alpha_{j}}\right)-\sum_{j=1}^{d}\left(z_{j}-\alpha_{j}\right)^{2}\right|}{\|z-\alpha\|^{2}} \\
& =\frac{16}{3} \frac{\left|\sum_{j=1}^{d}\left(z_{j}-\alpha_{j}\right)\left[\left(\overline{z_{j}}-\overline{\alpha_{j}}\right)-\left(z_{j}-\alpha_{j}\right)\right]\right|}{\|z-\alpha\|^{2}} \\
& \leq \frac{16}{3\|z-\alpha\|^{2}} \sum_{j=1}^{d}\left|z_{j}-\alpha_{j}\right|\left|\left(\overline{z_{j}}-z_{j}\right)+\left(\alpha_{j}-\overline{\alpha_{j}}\right)\right| \\
& \leq \frac{16}{3\|z-\alpha\|} \sum_{j=1}^{d}\left|2 i\left[\operatorname{Im}\left(\overline{z_{j}}\right)+\operatorname{Im}\left(\alpha_{j}\right)\right]\right| \\
& \leq \frac{32}{3\|z-\alpha\|} \sum_{j=1}^{d}\left(\left|\operatorname{Im}\left(\overline{z_{j}}\right)\right|+\left|\operatorname{Im}\left(\alpha_{j}\right)\right|\right) \\
& \leq \frac{32}{3\|z-\alpha\|} \sum_{j=1}^{d} 2 r \\
& =\frac{64 d r}{3\|z-\alpha\|} \text {. }
\end{aligned}
$$

Now, let

$$
M_{d}:=(0, \infty) \times(0, \infty) \times \mathbb{R}^{d} \times \mathbb{R}^{d} \times \mathrm{SO}(d) \subset \mathbb{R}^{q},
$$

where $q=2+\frac{1}{2} d(d+3)$. Note that $M_{d}$ is an open subset of $\mathbb{R}^{q}$. Given $e=\left(\lambda_{e}, r_{e}, a_{e}, b_{e}, A_{e}\right) \in$ $M_{d}$, set (cf. formulas (3.3) and (3.4))

$$
\varphi_{e}=\lambda_{e} A_{e} \circ i_{a_{e}, r_{e}}+b_{e} .
$$

More generally, put

$$
\mathbb{C} M_{d}:=\mathbb{C} \backslash\{0\} \times \mathbb{C} \backslash\{0\} \times \mathbb{C}^{d} \times \mathbb{C}^{d} \times \mathrm{SO}_{\mathbb{C}}(d) \subset \mathbb{C}^{q}
$$

Observe that $\mathbb{C} M_{d}$ is an open subset of $\mathbb{C}^{q}$, and for every $e \in \mathbb{C} M_{d}$ define $\varphi_{e}$ again as above (cf. formulas (3.5) and (3.6)).

Now, given $X$ a compact subset of $\mathbb{R}^{d}$ and $V \supset X$ an open subset of $\mathbb{R}^{d}$, as well as $s>0$, let

$$
M_{d}(X, V)_{s}=\left\{e \in M_{d}: a_{e} \in \mathbb{R}^{d} \backslash V \text { and } \sup _{x \in X}\left\|\varphi_{e}^{\prime}(x)\right\| \leq s\right\}
$$


Our last goal in this section is to prove Lemma 3.7, which will later be used to "complexify" the space of graph directed Markov systems. In order to prove it, we first establish two preliminary lemmas.

Lemma 3.5. Let $X$ be a compact subset of $\mathbb{R}^{d}$ and $V \supset X$ an open subset of $\mathbb{R}^{d}$. Then for every $\varepsilon>0$ and every $e \in M_{d}(X, V)_{1}$ there exists $\delta=\delta_{e}(\varepsilon)>0$ such that if $f \in$ $B_{\mathbb{C}^{q}}(e, \delta) \cap \mathbb{C} M_{d}$, then $\left\|\varphi_{f}-\varphi_{e}\right\|_{X} \leq \varepsilon$, where $\left\|\varphi_{f}-\varphi_{e}\right\|_{X}:=\sup _{x \in X}\left\|\varphi_{f}(x)-\varphi_{e}(x)\right\|$.

Proof. Fix $e \in M_{d}(X, V)_{1}$ and $0<\varepsilon<\min \{1,1 /(4 C(X, V))\}$, where $C(X, V)$ is the constant arising from Lemma 3.3. Set $\Delta:=\operatorname{dist}(X, \partial V)$ and $\tilde{\Delta}:=\Delta /(32 d)$. Take $\delta>0$ such that if $f \in B_{\mathbb{C}^{q}}(e, \delta) \cap \mathbb{C} M_{d}$, then

$$
\left\|a_{f}-a_{e}\right\| \leq \min \left\{\frac{\varepsilon}{16(1+3 d)}, \tilde{\Delta}\right\}
$$

$$
\left\|b_{f}-b_{e}\right\| \leq \frac{\varepsilon}{4}
$$

$$
\begin{gathered}
\left\|\lambda_{f} r_{f}^{2} A_{f}-\lambda_{e} r_{e}^{2} A_{e}\right\| \leq \Delta \frac{\varepsilon}{4}, \\
\left\|\lambda_{f} A_{f} a_{f}-\lambda_{e} A_{e} a_{e}\right\| \leq \frac{\varepsilon}{4}
\end{gathered}
$$

and

$$
\left|\lambda_{f}\right|\left|r_{f}\right|^{2}\left\|A_{f}\right\| \leq 2 \lambda_{e} r_{e}^{2}\left\|A_{e}\right\|^{2}=2 \lambda_{e} r_{e}^{2}
$$

Fix $f \in B_{\mathbb{C}^{q}}(e, \delta) \cap \mathbb{C} M_{d}$. Since $\left\|a_{f}-a_{e}\right\| \leq \tilde{\Delta}$, it follows from Lemma 3.2 that

$$
\varphi_{f}(x)=\lambda_{f} A_{f}\left(r_{f}^{2} \frac{x-a_{f}}{\|\| x-a_{f} \mid \|^{2}}+a_{f}\right)+b_{f}
$$


is well defined for all $x \in X$. Then for all $x \in X$ we have

$$
\begin{aligned}
& \left\|\varphi_{f}(x)-\varphi_{e}(x)\right\| \\
& =\left\|\lambda_{f} A_{f}\left(r_{f}^{2} \frac{x-a_{f}}{\left.\left\|x-a_{f}\right\|\right|^{2}}+a_{f}\right)+b_{f}-\lambda_{e} A_{e}\left(r_{e}^{2} \frac{x-a_{e}}{\left\|x-a_{e}\right\|^{2}}+a_{e}\right)-b_{e}\right\| \\
& =\left\|\begin{array}{l}
\left(\lambda_{f} r_{f}^{2} A_{f}-\lambda_{e} r_{e}^{2} A_{e}\right) \frac{x-a_{e}}{\left\|x-a_{e}\right\|^{2}}+\lambda_{f} r_{f}^{2} A_{f}\left(\frac{x-a_{f}}{\left\|x-a_{f}\right\|^{2}}-\frac{x-a_{e}}{\left\|x-a_{e}\right\|^{2}}\right) \\
+\left(\lambda_{f} A_{f} a_{f}-\lambda_{e} A_{e} a_{e}\right)+\left(b_{f}-b_{e}\right)
\end{array}\right\| \\
& \leq\left\|x-a_{e}\right\|^{-1} \cdot\left\|\lambda_{f} r_{f}^{2} A_{f}-\lambda_{e} r_{e}^{2} A_{e}\right\|+\left|\lambda_{f}\left\|\left.r_{f}\right|^{2}\right\| A_{f}\|\cdot\| \frac{x-a_{f}}{\left\|\left|x-a_{f} \|\right|^{2}\right.}-\frac{x-a_{e}}{\left\|x-a_{e}\right\|^{2}} \|\right. \\
& +\left\|\lambda_{f} A_{f} a_{f}-\lambda_{e} A_{e} a_{e}\right\|+\left\|b_{f}-b_{e}\right\| \\
& \leq \Delta^{-1} \cdot \Delta \frac{\varepsilon}{4}+\left|\lambda_{f}\left\|\left.r_{f}\right|^{2}\right\| A_{f} \| \frac{\|\| x-a_{e}\left\|^{2}\left(x-a_{f}\right)-\right\| x-a_{f}\left\|^{2}\left(x-a_{e}\right)\right\|}{\|\| x-a_{f}\left|\left\|^{2} \mid\right\| x-a_{e} \|^{2}\right.}\right. \\
& +\frac{\varepsilon}{4}+\frac{\varepsilon}{4} \\
& \leq \frac{3 \varepsilon}{4}+\left|\lambda_{f}\left\|\left.r_{f}\right|^{2}\right\| A_{f} \| \frac{\|\| x-a_{e}\left\|^{2}\left(x-a_{f}\right)-\right\| x-a_{f}\left\|^{2}\left(x-a_{e}\right)\right\|}{\left|\left\|x-a_{f}\left|\left\|\left.\right|^{2} \mid\right\| x-a_{e} \|^{2}\right.\right.\right.},\right.
\end{aligned}
$$

where we used (3.14), (3.15) and (3.16). Now

$$
\begin{aligned}
& \|\| x-a_{e}\left\|^{2}\left(x-a_{f}\right)-\right\| x-a_{f}\|\|^{2}\left(x-a_{e}\right) \| \\
& =\|\| x-a_{e}\left\|^{2}\left(a_{e}-a_{f}\right)+\left(\left\|x-a_{e}\right\|^{2}-\left.\left\|x-a_{f}\right\|\right|^{2}\right)\left(x-a_{e}\right)\right\| \\
& \leq\left\|a_{e}-a_{f}\right\| \cdot\left\|x-a_{e}\right\|^{2}+\left\|x-a_{e}\right\|\left|\left\|x-a_{e}\right\|^{2}-\left\|\left|x-a_{f}\right|\right\|^{2}\right| .
\end{aligned}
$$

Furthermore,

$$
\begin{aligned}
\left|\left\|x-a_{e}\right\|^{2}-\|\right| x-a_{f}||^{2} \mid & =\left|\sum_{j=1}^{d}\left(\left(x_{j}-a_{e_{j}}\right)^{2}-\left(x_{j}-a_{f_{j}}\right)^{2}\right)\right| \\
& =\left|\sum_{j=1}^{d}\left(a_{f_{j}}-a_{e_{j}}\right)\left(\left(x_{j}-a_{f_{j}}\right)+\left(x_{j}-a_{e_{j}}\right)\right)\right| \\
& \leq \sum_{j=1}^{d}\left|a_{f_{j}}-a_{e_{j}}\right|\left(\left|x_{j}-a_{f_{j}}\right|+\left|x_{j}-a_{e_{j}}\right|\right) \\
& \leq d\left\|a_{f}-a_{e}\right\|\left(\left\|x-a_{f}\right\|+\left\|x-a_{e}\right\|\right) .
\end{aligned}
$$


In virtue of (3.13) and Lemma 3.3 (recall that $0<\varepsilon<\min \{1,1 /(4 C(X, V))\}$ ), we have

$$
\frac{1}{2} \leq \frac{\left|\left\|\left|x-a_{f}\left\|\left.\right|^{2} \mid,\right\| x-a_{f} \|^{2}\right.\right.\right.}{\left\|x-a_{e}\right\|^{2}} \leq \frac{3}{2} .
$$

Combining this with (3.18) and (3.19), we get

$$
\begin{aligned}
\frac{\|\| x-a_{e}\left\|^{2}\left(x-a_{f}\right)-\right\| x-a_{f}\left\|^{2}\left(x-a_{e}\right)\right\|}{\|\| x-a_{f}\left\|^{2} \mid\right\| x-a_{e} \|^{2}} & \leq \frac{\left\|a_{f}-a_{e}\right\|}{\left|\left\|x-a_{f}\right\|\right|^{2} \mid}+\frac{d\left\|a_{f}-a_{e}\right\|\left(\left\|x-a_{f}\right\|+\left\|x-a_{e}\right\|\right)}{\|\| x-a_{f}\left\|\left.\right|^{2} \mid\right\| x-a_{e} \|} \\
& \leq \frac{\left\|a_{f}-a_{e}\right\|}{(1 / 2)\left\|x-a_{e}\right\|^{2}}+d\left\|a_{f}-a_{e}\right\| \frac{3\left\|x-a_{e}\right\|}{(1 / 2)\left\|x-a_{e}\right\|^{3}} \\
& =2(1+3 d) \frac{\left\|a_{f}-a_{e}\right\|}{\left\|x-a_{e}\right\|^{2}} .
\end{aligned}
$$

Since $\left\|\varphi_{e}^{\prime}(x)\right\| \leq 1$, we get from (3.4) that $\lambda_{e} r_{e}^{2} \leq\left\|x-a_{e}\right\|^{2}$. Using this, (3.17), the above inequality and (3.13), we deduce that

$$
\begin{aligned}
\left|\lambda_{f}\left\|\left.r_{f}\right|^{2}\right\| A_{f} \| \frac{\|\| x-a_{e}\left\|^{2}\left(x-a_{f}\right)-\right\| x-a_{f}\left\|\left.\right|^{2}\left(x-a_{e}\right)\right\|}{\|\| x-a_{f}\left\|^{2} \mid\right\| x-a_{e} \|^{2}}\right. & \leq 2 \lambda_{e} r_{e}^{2} \cdot 2(1+3 d) \frac{\left\|a_{f}-a_{e}\right\|}{\left\|x-a_{e}\right\|^{2}} \\
& \leq 2\left\|x-a_{e}\right\|^{2} \cdot 2(1+3 d) \frac{\left\|a_{f}-a_{e}\right\|}{\left\|x-a_{e}\right\|^{2}} \\
& =4(1+3 d)\left\|a_{f}-a_{e}\right\| \\
& \leq \varepsilon / 4 .
\end{aligned}
$$

We thus conclude that $\left\|\varphi_{f}(x)-\varphi_{e}(x)\right\|<\varepsilon$. Since this is true for every $x \in X$, we deduce that $\left\|\varphi_{f}-\varphi_{e}\right\|_{X} \leq \varepsilon$. Finally, because $f$ was chosen arbitrarily in $B_{\mathbb{C}^{q}}(e, \delta) \cap \mathbb{C} M_{d}$, the proof is complete.

Here is the second preliminary lemma.

Lemma 3.6. Let $X$ be a compact subset of $\mathbb{R}^{d}$ and $V \supset X$ an open subset of $\mathbb{R}^{d}$. Fix $s \in(0,1]$. Then for every $\varepsilon>0$ there exists $r>0$ such that for all $e \in M_{d}(X, V)_{s}$ there is $\delta=\delta_{e}>0$ such that if $f \in B_{\mathbb{C}^{q}}(e, \delta) \cap \mathbb{C}_{d}$, then $\left\|\varphi_{f}^{\prime}(z)\right\| \leq(1+\varepsilon)$ s for all $z \in B_{\mathbb{C}^{d}}(X, r)$.

Proof. For every $g \in \mathbb{C} M_{d}$ and every $z \in \mathbb{C}^{d} \backslash Z_{a_{g}}$ define the matrix $\left(\hat{g}_{i j}(z)\right)_{1 \leq i, j \leq d}$ by the formula

$$
\hat{g}_{i j}(z)=\frac{\left(z_{i}-a_{g_{i}}\right)\left(z_{j}-a_{g_{j}}\right)}{\|\| z-a_{g} \mid \|^{2}} .
$$

Let $\varepsilon \in(0,1 / 2)$. Let $\tilde{\varepsilon} \in(0, \varepsilon]$ be such that $(1+\tilde{\varepsilon})^{2}(1-\tilde{\varepsilon})^{-1}(1+12 d \tilde{\varepsilon}) \leq 1+\varepsilon$. Let $\Delta:=\operatorname{dist}(X, \partial V)$ and take $0<r \leq 3 \Delta \tilde{\varepsilon} /(128 d)$ such that, according to Lemma 3.3,

$$
\max \left\{\left|\frac{\|z-\alpha\|^{2}}{\|w-\beta\|^{2}}-1\right|,\left|\frac{\left\||z-\alpha \||^{2}\right.}{\||w-\beta|\|^{2}}-1\right|\right\} \leq \tilde{\varepsilon}<1 / 2
$$


for every $(z, \alpha),(w, \beta) \in B_{\mathbb{C}^{d}}(X, r) \times B_{\mathbb{C}^{d}}\left(\mathbb{R}^{d} \backslash V, r\right)$. Since $0<r<\tilde{\Delta}:=\Delta /(32 d)$, Lemma 3.4 gives

$$
\left|\frac{\|z-\alpha\|^{2}}{\|\| z-\alpha \|^{2}}-1\right| \leq \frac{64 d r}{3\|z-\alpha\|} \leq \frac{64 d r}{3(\Delta-2 r)} \leq \frac{128 d r}{3 \Delta} \leq \tilde{\varepsilon} .
$$

for every couple $(z, \alpha) \in B_{\mathbb{C}^{d}}(X, r) \times B_{\mathbb{C}^{d}}\left(\mathbb{R}^{d} \backslash V, r\right)$.

Now, let $e \in M_{d}(X, V)_{s}$. Take $0<\delta \leq r$ such that if $f \in B_{\mathbb{C} q}(e, \delta) \cap \mathbb{C} M_{d}$, then $\left|\lambda_{f}\right|\left|r_{f}\right|^{2} \leq(1+\tilde{\varepsilon}) \lambda_{e} r_{e}^{2}$ and $\left\|A_{f}\right\| \leq(1+\tilde{\varepsilon})\left\|A_{e}\right\|=1+\tilde{\varepsilon}$. Let $f \in B_{\mathbb{C}^{q}}(e, \delta) \cap \mathbb{C} M_{d}$. Fix $1 \leq i, j \leq d$ and $z \in B_{\mathbb{C}^{d}}(X, r)$. Then there exists $x \in X$ such that $\|z-x\|<r$. We thereafter have

$$
\begin{aligned}
&\left|\hat{f}_{i j}(z)-\hat{e}_{i j}(x)\right|= \mid\left(\left(x_{i}-a_{e_{i}}\right)\left(x_{j}-a_{e_{j}}\right)-\left(z_{i}-a_{f_{i}}\right)\left(z_{j}-a_{f_{j}}\right)\right)\left\|x-a_{e}\right\|^{-2}+ \\
&\left(\left\|x-a_{e}\right\|^{-2}-\left|\left\|z-a_{f}|\||^{-2}\right)\left(z_{i}-a_{f_{i}}\right)\left(z_{j}-a_{f_{j}}\right)\right|\right. \\
& \leq\left\|x-a_{e}\right\|^{-2}\left|\left(x_{i}-a_{e_{i}}\right)\left(x_{j}-a_{e_{j}}\right)-\left(z_{i}-a_{f_{i}}\right)\left(z_{j}-a_{f_{j}}\right)\right|+ \\
&\left\|z-a_{f}\right\|^{2}\left|\left\|x-a_{e}\right\|^{-2}-\right|\left\|z-a_{f}\right\|^{-2} \mid \\
& \leq\left\|x-a_{e}\right\|^{-2}\left|\left(x_{i}-a_{e_{i}}\right)\left(\left(x_{j}-z_{j}\right)+\left(a_{f_{j}}-a_{e_{j}}\right)\right)+\left(z_{j}-a_{f_{j}}\right)\left(\left(x_{i}-z_{i}\right)+\left(a_{f_{i}}-a_{e_{i}}\right)\right)\right| \\
& \quad+\left|\frac{\left\|z-a_{f}\right\|^{2}}{\left\|x-a_{e}\right\|^{2}}-\frac{\left\|z-a_{f}\right\|^{2}}{\|\left.\left|a_{f}\right|\right|^{2}}\right| \\
& \leq \\
& \quad\left\|x-a_{e}\right\|^{-2}\left[\left\|x-a_{e}\right\|\left(\|x-z\|+\left\|a_{f}-a_{e}\right\|\right)+\left\|z-a_{f}\right\|\left(\|x-z\|+\left\|a_{f}-a_{e}\right\|\right)\right] \\
& \quad+\left|\frac{\left\|z-a_{f}\right\|^{2}}{\left\|x-a_{e}\right\|^{2}}-1\right|+\left|\frac{\left\|z-a_{f}\right\|^{2}}{\|\| z-a_{f} \|^{2}}-1\right| \\
& \leq\left\|x-a_{e}\right\|^{-2}\left(\left\|x-a_{e}\right\|+\left\|z-a_{f}\right\|\right)(r+\|f-e\|)+2 \tilde{\varepsilon}
\end{aligned}
$$

where we successively used (3.20) with $\alpha=a_{f}, w=x$ and $\beta=a_{e}$, and (3.21) with $\alpha=a_{f}$, keeping in mind that $a_{e} \in \mathbb{R}^{d} \backslash V$ since $e \in M_{d}(X, V)_{s}$. Since $\delta \leq r$ and $f \in B_{\mathbb{C}^{q}}(e, \delta) \cap \mathbb{C} M_{d}$, we obtain

$$
\left|\hat{f}_{i j}(z)-\hat{e}_{i j}(x)\right| \leq\left\|x-a_{e}\right\|^{-2}\left(\left\|x-a_{e}\right\|+\left\|z-a_{f}\right\|\right) \cdot 2 r+2 \tilde{\varepsilon} .
$$

Using (3.20) again, we deduce that

$$
\begin{aligned}
\left|\hat{f}_{i j}(z)-\hat{e}_{i j}(x)\right| & \leq\left\|x-a_{e}\right\|^{-2}\left(\left\|x-a_{e}\right\|+2\left\|x-a_{e}\right\|\right) \cdot 2 r+2 \tilde{\varepsilon} \\
& =6 r\left\|x-a_{e}\right\|^{-1}+2 \tilde{\varepsilon} \leq 6 \Delta^{-1} r+2 \tilde{\varepsilon}<3 \tilde{\varepsilon} .
\end{aligned}
$$

Since the above argument is in particular valid with $f$ replaced by $e$, we have

$$
\begin{aligned}
\left|\hat{f}_{i j}(z)-\hat{e}_{i j}(z)\right| & \leq\left|\hat{f}_{i j}(z)-\hat{e}_{i j}(x)\right|+\left|\hat{e}_{i j}(x)-\hat{e}_{i j}(z)\right| \\
& <3 \tilde{\varepsilon}+3 \tilde{\varepsilon}=6 \tilde{\varepsilon} .
\end{aligned}
$$

Hence $\|\hat{f}-\hat{e}\|<6 d \tilde{\varepsilon}$. Now, the derivative matrix $\varphi_{f}^{\prime}(z)$ of $\varphi_{f}$, written as the matrix of partial derivatives in complex coordinates at a point $z \in \mathbb{C}^{d}$, is equal to $\lambda_{f} r_{f}^{2} \mid \| z-$ 
$a_{f}||^{-2} A_{f}(I d-2 \hat{f})(z)$. By our choice of $\delta$ we have $\left|\lambda_{f} \| r_{f}\right|^{2} \leq(1+\tilde{\varepsilon}) \lambda_{e} r_{e}^{2}$ and $\left\|A_{f}\right\| \leq$ $(1+\tilde{\varepsilon})\left\|A_{e}\right\|=1+\tilde{\varepsilon}$. Moreover, $\|(I d-2 \hat{f})-(I d-2 \hat{e})\|=2\|\hat{f}-\hat{e}\|<12 d \tilde{\varepsilon}$. Using once again (3.20) as well as the above estimates, we get

$$
\begin{aligned}
\left\|\varphi_{f}^{\prime}(z)\right\| & \leq(1+\tilde{\varepsilon}) \lambda_{e} r_{e}^{2} \cdot(1-\tilde{\varepsilon})^{-1}\left\|x-a_{e}\right\|^{-2} \cdot(1+\tilde{\varepsilon}) \cdot(1+12 d \tilde{\varepsilon}) \\
& =(1+\tilde{\varepsilon})^{2}(1-\tilde{\varepsilon})^{-1}(1+12 d \tilde{\varepsilon}) \lambda_{e} r_{e}^{2}\left\|x-a_{e}\right\|^{-2} \\
& \leq(1+\varepsilon) \lambda_{e} r_{e}^{2}\left\|x-a_{e}\right\|^{-2} \leq(1+\varepsilon) s .
\end{aligned}
$$

Since this is true for every $z \in B_{\mathbb{C}^{d}}(x, r)$, the result follows.

Now, given two compact subsets $X_{1}, X_{2}$ of $\mathbb{R}^{d}$, two open subsets $V_{1}, V_{2}$ of $\mathbb{R}^{d}$ containing $X_{1}$ and $X_{2}$ respectively, and $s \in(0,1)$, we denote by $\mathbb{R}_{s}\left(\left(X_{1}, V_{1}\right) ;\left(X_{2}, V_{2}\right)\right)$ the subset of $M_{d}$ consisting of all $e$ such that

$$
\varphi_{e}\left(X_{1}\right) \subset X_{2}, \quad \varphi_{e}\left(V_{1}\right) \subset V_{2}
$$

and

$$
\sup \left\{\left\|\varphi_{e}^{\prime}(x)\right\|: x \in X_{1}\right\} \leq s .
$$

Note that $\mathbb{R}_{s}\left(\left(X_{1}, V_{1}\right) ;\left(X_{2}, V_{2}\right)\right) \subset M_{d}\left(X_{1}, V_{1}\right)_{s}$. We define analogously the complex subset $\mathbb{C}_{s}\left(\left(X_{1}, V_{1}\right) ;\left(X_{2}, V_{2}\right)\right)$ of $\mathbb{C}_{d}$, requiring this time that the $X_{i}$ 's be compact subsets of $\mathbb{C}^{d}$ and that the $V_{i}$ 's be open subsets of $\mathbb{C}^{d}$, each containing the corresponding $X_{i}$.

Lemma 3.7. Suppose that $X_{1}$ and $X_{2}$ are compact subsets of $\mathbb{R}^{d}$, and that $V_{1}$ and $V_{2}$ are open subsets of $\mathbb{R}^{d}$ containing $X_{1}$ and $X_{2}$, respectively. Fix $s \in(0,1)$. Then there exists a constant $R:=R_{s}\left(\left(X_{1}, V_{1}\right) ;\left(X_{2}, V_{2}\right)\right)>0$ such that for every $0<\tilde{R} \leq R$, each $e \in \mathbb{R}_{s}\left(\left(X_{1}, V_{1}\right) ;\left(X_{2}, V_{2}\right)\right)$ admits $\delta_{e}(\tilde{R})>0$ such that

$$
B_{\mathbb{C}^{q}}\left(e, \delta_{e}(\tilde{R})\right) \cap \mathbb{C} M_{d} \subset \mathbb{C}_{\frac{s+1}{2}}\left(\left(\bar{B}_{\mathbb{C}^{d}}\left(X_{1}, \tilde{R}\right), B_{\mathbb{C}^{d}}\left(X_{1}, 2 \tilde{R}\right)\right) ;\left(\bar{B}_{\mathbb{C}^{d}}\left(X_{2}, \tilde{R}\right), B_{\mathbb{C}^{d}}\left(X_{2}, 2 \tilde{R}\right)\right)\right) .
$$

Proof. Apply Lemma 3.6 with $X=X_{1}, V=V_{1}$ and $\varepsilon>0$ so small that

$$
(1+\varepsilon) s \leq \frac{s+1}{2} \text {. }
$$

Let $r>0$ be the corresponding number mentioned in that lemma. Fix an element $e \in$ $\mathbb{R}_{s}\left(\left(X_{1}, V_{1}\right) ;\left(X_{2}, V_{2}\right)\right) \subset M_{d}\left(X_{1}, V_{1}\right)_{s}$ and denote by $\delta_{e, 1}>0$ the corresponding number $\delta$ produced in Lemma 3.6. The lemma then asserts that

$$
\left\|\varphi_{f}^{\prime}(z)\right\| \leq \frac{s+1}{2}
$$

for all $z \in B_{\mathbb{C}^{d}}\left(X_{1}, r\right)$ and all $f \in B_{\mathbb{C}^{q}}\left(e, \delta_{e, 1}\right) \cap \mathbb{C} M_{d}$. Now, fix $0<\tilde{r} \leq r$ and apply Lemma 3.5 with

$$
\varepsilon=\frac{1-s}{4} \tilde{r} .
$$

Denote the resulting $\delta>0$ by $\delta_{e, 2}(\tilde{r})$. Lemma 3.5 then states that

$$
\left\|\varphi_{f}-\varphi_{e}\right\|_{X_{1}} \leq \frac{1-s}{4} \tilde{r}
$$


for all $f \in B_{\mathbb{C}^{q}}\left(e, \delta_{e, 2}(\tilde{r})\right) \cap \mathbb{C} M_{d}$. Now, set $\delta_{e}(\tilde{r}):=\min \left\{\delta_{e, 1}, \delta_{e, 2}(\tilde{r})\right\}$. Consider an arbitrary radius $\eta \in[\tilde{r} / 2, \tilde{r}]$ and take any point $z \in B_{\mathbb{C}^{d}}\left(X_{1}, \eta\right)$. Then there exists $x \in X_{1}$ such that $\|z-x\|<\eta$. Using (3.24) and (3.25), we get that for all $f \in B_{\mathbb{C}^{q}}\left(e, \delta_{e}(\tilde{r})\right) \cap \mathbb{C} M_{d}$

$$
\begin{aligned}
\left\|\varphi_{f}(z)-\varphi_{e}(x)\right\| & \leq\left\|\varphi_{f}(z)-\varphi_{f}(x)\right\|+\left\|\varphi_{f}(x)-\varphi_{e}(x)\right\| \\
& \leq \frac{s+1}{2}\|z-x\|+\frac{1-s}{4} \tilde{r}<\frac{s+1}{2} \eta+\frac{1-s}{4} \tilde{r} \leq \eta .
\end{aligned}
$$

Since $\varphi_{e}(x) \in X_{2}$, we deduce that $\varphi_{f}(z) \in B_{\mathbb{C}^{d}}\left(X_{2}, \eta\right)$. Consequently, $\varphi_{f}\left(B_{\mathbb{C}^{d}}\left(X_{1}, \eta\right)\right) \subset$ $B_{\mathbb{C}^{d}}\left(X_{2}, \eta\right)$ and $\varphi_{f}\left(\bar{B}_{\mathbb{C}^{d}}\left(X_{1}, \eta\right)\right) \subset \bar{B}_{\mathbb{C}^{d}}\left(X_{2}, \eta\right)$. Looking at the first of these inclusions with $\eta=\tilde{r} / 2$, at the latter one with $\eta=\tilde{r}$, and considering (3.24), we conclude that

$$
B_{\mathbb{C}^{q}}\left(e, \delta_{e}(\tilde{r} / 2)\right) \cap \mathbb{C} M_{d} \subset \mathbb{C}_{\frac{s+1}{2}}\left(\left(\bar{B}_{\mathbb{C}^{d}}\left(X_{1}, \tilde{r} / 2\right), B_{\mathbb{C}^{d}}\left(X_{1}, \tilde{r}\right)\right) ;\left(\bar{B}_{\mathbb{C}^{d}}\left(X_{2}, \tilde{r} / 2\right), B_{\mathbb{C}^{d}}\left(X_{2}, \tilde{r}\right)\right)\right) .
$$

Setting $R=r / 2$ and $\tilde{R}=\tilde{r} / 2$ completes the proof.

\section{Symbolic Dynamics}

In this section we collect the fundamental ergodic (thermodynamic formalism) results in one-sided symbolic dynamics. All of them can be found with proofs in [4], [5] or [9]. Let $I$ be a countable set and let $A: I \times I \rightarrow\{0,1\}$ be a matrix, often called incidence matrix. A finite or countably infinite tuple (commonly called word) $\omega$ of elements of $I$ is called $A$-admissible if $A_{a b}=1$ for any two consecutive elements $a, b$ of $\omega$. The matrix $A$ is said to be finitely irreducible provided that there exists a finite set $F$ of finite $A$-admissible words such that for any two elements $a, b$ of $I$ there is a word $\gamma$ in $F$ such that the word $a \gamma b$ is $A$-admissible. Throughout the entire section the matrix $A$ is assumed to be finitely irreducible. We denote the set of all countably infinite $A$-admissible words by

$$
I_{A}^{\infty}=\left\{\left(\omega_{n}\right)_{n=1}^{\infty} \in I^{\infty}: \forall(n \in \mathbb{N}), A_{\omega_{n} \omega_{n+1}}=1\right\} .
$$

Obviously $I_{A}^{\infty}$ is a closed subset of $I^{\infty}$ when this latter is endowed with the product topology, and we equip the former with the topology inherited from the latter. We further denote by $I_{A}^{n}$ the set of all $A$-admissible words of length $n \geq 1$, and by $I_{A}^{*}$ the set of all finite $A$-admissible words. Recall that the left shift map $\sigma: I^{\infty} \rightarrow I^{\infty}$, which by definition drops the first entry of $\omega$, is defined by the formula

$$
\sigma\left(\left(\omega_{n}\right)_{n=1}^{\infty}\right)=\left(\omega_{n+1}\right)_{n=1}^{\infty}
$$

This shift is clearly continuous, and of course $\sigma\left(I_{A}^{\infty}\right) \subset I_{A}^{\infty}$. Given $\omega \in I_{A}^{*}$ we denote by $|\omega|$ the length of $\omega$, that is, the unique $n \geq 1$ such that $\omega \in I_{A}^{n}$. If $\omega \in I_{A}^{\infty}$ and $n \geq 1$, then we will use the notation

$$
\left.\omega\right|_{n}:=\omega_{1} \ldots \omega_{n} .
$$

We will also denote the empty word by $\varepsilon$ and declare that its length is 0 . For $\omega, \tau \in I_{A}^{\infty}$, we define $\omega \wedge \tau \in I_{A}^{\infty} \cup I_{A}^{*} \cup\{\varepsilon\}$ as the longest initial subword common to $\omega$ and $\tau$. Finally, for every finite $A$-admissible word $\omega=\omega_{1} \ldots \omega_{n}$, set

$$
[\omega]=\left\{\tau \in I_{A}^{\infty}:\left.\tau\right|_{n}=\omega\right\} .
$$

The set $[\omega]$ is called the cylinder generated by $\omega$. 
Let us now talk about functions, also called potentials, on $I_{A}^{\infty}$. Given $s \in(0,1)$, recall that a function $\psi: I_{A}^{\infty} \rightarrow \mathbb{C}$ is $s$-Hölder continuous if

$$
v_{s}(\psi):=\sup _{n \geq 1} v_{s, n}(\psi)<\infty,
$$

where

$$
v_{s, n}(\psi)=\sup \left\{\frac{|\psi(\omega)-\psi(\tau)|}{s^{n}}: \omega, \tau \in I_{A}^{\infty} \text { and }|\omega \wedge \tau| \geq n\right\} .
$$

We will denote by $\mathcal{H}_{s}$ the set of all $s$-Hölder continuous functions on $I_{A}^{\infty}$. Recall also that a function $\psi: I_{A}^{\infty} \rightarrow \mathbb{C}$ is said to be summable if

$$
\sum_{i \in I} \exp \left(\sup \left(\left.\operatorname{Re} \psi\right|_{[i]}\right)\right)<\infty .
$$

We henceforth denote by $\mathcal{K}_{s}$ the set of all $s$-Hölder continuous, summable functions.

Now, let $\psi: I_{A}^{\infty} \rightarrow \mathbb{C}$ be a continuous function. Let also $M_{\sigma}$ denote the set of all shiftinvariant Borel probability measures on $I_{A}^{\infty}$. A measure $\mu \in M_{\sigma}$ is said to be a Gibbs state for $\psi$ provided that there are constants $C \geq 1$ and $P \in \mathbb{R}$ such that

$$
C^{-1} \leq \frac{\mu([\omega])}{\exp \left(\operatorname{Re} S_{n} \psi(\tau)-P n\right)} \leq C
$$

for all $n \geq 1$, all $\omega \in I_{A}^{n}$ and all $\tau \in[\omega]$. Note that if $\psi$ has a Gibbs state, then $\psi$ is summable. The topological pressure $\mathrm{P}(\psi)$ is defined as follows:

$$
\mathrm{P}(\psi)=\lim _{n \rightarrow \infty} \frac{1}{n} \log \sum_{\omega \in I_{A}^{n}} \exp \left(\sup \left(\left.\operatorname{Re} S_{n} \psi\right|_{[\omega]}\right)\right) .
$$

It follows from (4.1) that if $\psi$ admits a Gibbs state, then $P=\mathrm{P}(\psi)$. The proofs of the following two fundamental results can be found in [4], [5] or [9].

Theorem 4.1. (Existence and Uniqueness of Gibbs States) For every Hölder continuous, summable potential $\psi: I_{A}^{\infty} \rightarrow \mathbb{C}$ there exists a unique Gibbs state $\mu_{\psi}$ on $I_{A}^{\infty}$. Furthermore, this state is ergodic.

Theorem 4.2. (Variational Principle) For every Hölder continuous, summable potential $\psi: I_{A}^{\infty} \rightarrow \mathbb{C}$ we have

$\sup \left\{h_{\mu}(\sigma)+\int_{I_{A}^{\infty}} \operatorname{Re} \psi d \mu: \mu \in M_{\sigma}\right.$ and $\left.\int \operatorname{Re} \psi d \mu>-\infty\right\}=\mathrm{P}(\psi)=h_{\mu_{\psi}}(\sigma)+\int_{I_{A}^{\infty}} \operatorname{Re} \psi d \mu_{\psi}$ where $\mu_{\psi}$ is the unique Gibbs state for $\psi$, and is the only measure at which the supremum is taken on.

Any measure that realizes the supremum value in the above variational principle is called an equilibrium state for $\psi$. With this terminology, Theorem 4.2 can be reformulated as follows.

Theorem 4.3. If $\psi: I_{A}^{\infty} \rightarrow \mathbb{C}$ is a Hölder continuous, summable potential, then the unique Gibbs state $\mu_{\psi}$ for $\psi$ is the unique equilibrium state for $\psi$. 
We will in a moment define the Perron-Frobenius operator $\mathcal{L}_{\psi}: C_{b}\left(I_{A}^{\infty}\right) \rightarrow C_{b}\left(I_{A}^{\infty}\right)$, where $C_{b}\left(I_{A}^{\infty}\right)$ represents the space of all bounded, continuous complex-valued functions on $I_{A}^{\infty}$. But first, given $i \in I$ and $g: I_{A}^{\infty} \rightarrow \mathbb{C}$, define the mapping $g \circ i: I_{A}^{\infty} \rightarrow \mathbb{C} \cup\{-\infty\}$ by the formula

if $g \in \mathcal{K}_{s}$, and

$$
g \circ i(\omega)=\left\{\begin{array}{ccc}
g(i \omega) & \text { if } & A_{i \omega_{1}}=1 \\
-\infty & \text { if } & A_{i \omega_{1}}=0
\end{array}\right.
$$

$$
g \circ i(\omega)=\left\{\begin{array}{lll}
g(i \omega) & \text { if } & A_{i \omega_{1}}=1 \\
0 & \text { if } & A_{i \omega_{1}}=0
\end{array}\right.
$$

otherwise. Now fix $\psi \in \mathcal{K}_{s}$ and notice that for every $g \in C_{b}\left(I_{A}^{\infty}\right)$, the operator

$$
\mathcal{L}_{\psi}(g)=\sum_{i \in I} e^{\psi \circ i} g \circ i
$$

is well defined (with the convention that $e^{-\infty}=0,0 \cdot(-\infty)=0$ and $-\infty-(-\infty)=0$ ), belongs to $C_{b}\left(I_{A}^{\infty}\right)$ and $\left\|\mathcal{L}_{\psi}(g)\right\|_{\infty} \leq \sum_{i \in I} \exp \left(\sup \left(\left.\operatorname{Re} \psi\right|_{[i]}\right)\right)\|g\|_{\infty}$. We have thus defined the operator $\mathcal{L}_{\psi}$ acting continuously on $C_{b}\left(I_{A}^{\infty}\right)$ with

$$
\left\|\mathcal{L}_{\psi}\right\|_{\infty} \leq \sum_{i \in I} \exp \left(\operatorname{Re} \sup \left(\left.\psi\right|_{[i]}\right)\right)<\infty .
$$

In fact, as proved in [4], $\mathcal{L}_{\psi}$ preserves the Banach space $\mathcal{H}_{s}^{b}:=\mathcal{H}_{s} \cap C_{b}\left(I_{A}^{\infty}\right)$ when this latter is endowed with the $s$-norm, namely $\|g\|_{s}:=\|g\|_{\infty}+v_{s}(g)$, where $g \in \mathcal{H}_{s}^{b}$. The operator $\mathcal{L}_{\psi}$ is called Perron-Frobenius operator. As an immediate consequence of Theorem 2.4.6 from [4], we get the following:

Theorem 4.4. If $\psi \in \mathcal{K}_{s}$, then $e^{\mathrm{P}(\psi)}$ is a simple isolated eigenvalue of the Perron-Frobenius operator $\mathcal{L}_{\psi}: \mathcal{H}_{s}^{b} \rightarrow \mathcal{H}_{s}^{b}$.

One of the key facts needed to prove the real analyticity of Hausdorff dimension is Theorem 3.8 from [3]:

Theorem 4.5. Let $\Gamma \subset \mathbb{C}$ be an open subset of $\mathbb{C}$, and suppose that the function $\gamma \mapsto \psi_{\gamma} \in$ $\mathcal{K}_{s}, \gamma \in \Gamma$, is continuous. If the function $\gamma \mapsto \psi_{\gamma}(\omega) \in \mathbb{C}, \gamma \in \Gamma$, is holomorphic for every $\omega \in I_{A}^{\infty}$, then the function $\gamma \mapsto \mathcal{L}_{\psi_{\gamma}} \in L\left(\mathcal{H}_{s}^{b}\right)$ is also holomorphic.

\section{Graph Directed Markov Systems: Preliminaries}

In this section we begin our study of graph directed Markov systems. Let us recall the definition of these systems (see also [4]). Graph directed Markov systems are based upon a directed multigraph and an associated incidence matrix, $(V, I, i n, t o, A)$. The multigraph consists of a finite set $V$ of vertices and a countable (either finite or infinite) set of directed edges $I$ and two functions in, to $: I \rightarrow V$. For each edge $i \in I$, in $(i)$ is the initial vertex of that edge and to $(i)$ its terminal vertex. The edge goes from in $(i)$ to to $(i)$. Also, an incidence matrix $A: I \times I \rightarrow\{0,1\}$ is given. That matrix is an edge incidence matrix: it determines which edges may follow a given edge. And it does so while respecting the multigraph, that is it has the property that if $A_{u v}=1$, then $t o(u)=i n(v)$. We will consider finite and infinite walks through the vertex set which are consistent with the incidence matrix. That 
is, adopting the notation from section 4 , we denote the set of infinite $A$-admissible words $I_{A}^{\infty}$ on the alphabet $I$ by

$$
I_{A}^{\infty}=\left\{\omega \in I^{\infty}: A_{\omega_{n} \omega_{n+1}}=1 \text { for all } n \geq 1\right\} .
$$

A Graph Directed Markov System (GDMS) consists of a directed multigraph and an associated incidence matrix, $(V, I, i n, t o, A)$, together with a set of non-empty compact metric spaces $\left\{X_{v}\right\}_{v \in V}$, a number $s, 0<s<1$, and for every $i \in I$, a one-to-one contraction $\varphi_{i}: X_{t o(i)} \rightarrow X_{i n(i)}$ with a Lipschitz constant less than or equal to $s$. Briefly, the set

$$
\Phi=\left\{\varphi_{i}: X_{t o(i)} \rightarrow X_{i n(i)}\right\}_{i \in I}
$$

is called a GDMS. We now describe its limit set. For each $\omega \in I_{A}^{*}$, say $\omega \in I_{A}^{n}$, we consider the map coded by $\omega$,

$$
\varphi_{\omega}:=\varphi_{\omega_{1}} \circ \cdots \circ \varphi_{\omega_{n}}: X_{t o\left(\omega_{n}\right)} \rightarrow X_{i n\left(\omega_{1}\right)} .
$$

For each $\omega \in I_{A}^{\infty}$, the sets $\left(\varphi_{\left.\omega\right|_{n}}\left(X_{t o\left(\omega_{n}\right)}\right)\right)_{n=1}^{\infty}$ form a descending sequence of non-empty compact sets and therefore $\bigcap_{n=1}^{\infty} \varphi_{\left.\omega\right|_{n}}\left(X_{t o\left(\omega_{n}\right)}\right) \neq \emptyset$. Since for every $n \geq 1$ we have $\operatorname{diam}\left(\varphi_{\left.\omega\right|_{n}}\left(X_{t o\left(\omega_{n}\right)}\right)\right) \leq s^{n} \operatorname{diam}\left(X_{t o\left(\omega_{n}\right)}\right) \leq s^{n} \max \left\{\operatorname{diam}\left(X_{v}\right): v \in V\right\}$, we conclude that the intersection

$$
\bigcap_{n=1}^{\infty} \varphi_{\left.\omega\right|_{n}}\left(X_{t o\left(\omega_{n}\right)}\right)
$$

is a singleton and we denote its element by $\pi_{\Phi}(\omega)$. In this way we have defined the coding map $\pi_{\Phi}$ from $I_{A}^{\infty}$ to $X:=\bigoplus_{v \in V} X_{v}$, the disjoint union of the compact sets $X_{v}$. The set

$$
J_{\Phi}=\pi_{\Phi}\left(I_{A}^{\infty}\right)
$$

is the limit set of the GDMS $\Phi$.

A GDMS is called conformal (and thus is a CGDMS) if the following conditions are satisfied.

(4a) For every vertex $v \in V, X_{v}$ is a compact connected subset of a Euclidean space $\mathbb{R}^{d}$ (the dimension $d \geq 1$ being common to all $v \in V$ ) and $X_{v}=\overline{\operatorname{Int}\left(X_{v}\right)}$.

(4b) Open Set Condition $(O S C)$ : For all $a, b \in I, a \neq b$,

$$
\varphi_{a}\left(\operatorname{Int}\left(X_{t o(a)}\right)\right) \cap \varphi_{b}\left(\operatorname{Int}\left(X_{t o(b)}\right)\right)=\emptyset .
$$

(4c) For every vertex $v \in V$ there exists an open connected set $W_{v} \supset X_{v}$ such that for every $i \in I$ with to $(i)=v$, the map $\varphi_{i}$ extends to a $C^{1}$ conformal diffeomorphism of $W_{v}$ into $W_{\text {in }(i)}$.

(4d) Cone Property: There exist $\gamma, l>0$ such that for every $x \in X \subset \mathbb{R}^{d}$ there exists an open cone $\operatorname{Con}(x, \gamma, l) \subset \operatorname{Int}(X)$ with vertex $x$, central angle of measure $\gamma$, and altitude $l$.

(4e) There are two constants $L \geq 1$ and $\alpha>0$ such that

$$
\left\|\varphi_{i}^{\prime}(y)\right\|-\left\|\varphi_{i}^{\prime}(x)\right\| \mid \leq L\left\|\left(\varphi_{i}^{\prime}\right)^{-1}\right\|^{-1}\|y-x\|^{\alpha}
$$

for every $i \in I$ and every pair of points $x, y \in X_{t o(i)}$, where $\left\|\varphi_{i}^{\prime}(x)\right\|$ means the norm of the derivative of $\varphi$ at $x$. 
A GDMS $\Phi$ which satisfies (4a) and (4c) - (4e) without necessarily satisfying (4b) will be called a WPO CGDMS, where WPO stands for "With Potential Overlaps". Obviously, a CGDMS is a WPO CGDMS without overlaps.

The following remarkable result was proved in [4].

Proposition 5.1. If $d \geq 2$ and a GDMS $\Phi=\left\{\varphi_{i}\right\}_{i \in I}$ satisfies conditions (4a) and (4c), then it also satisfies condition (4e) with $\alpha=1$.

As shown in [4], the following result is a rather straightforward consequence of (4e).

Lemma 5.2. If $d \geq 2$ and a GDMS $\Phi=\left\{\varphi_{i}\right\}_{i \in I}$ satisfies conditions (4a) and (4c) (and thus (4e)), then for all $\omega \in I_{A}^{*}$ and all $x, y \in W_{\text {to }(\omega)}$, we have

$$
\left|\log \left\|\varphi_{\omega}^{\prime}(y)\right\|-\log \left\|\varphi_{\omega}^{\prime}(x)\right\|\right| \leq \frac{L}{1-\alpha}\|y-x\|^{\alpha} .
$$

As an immediate consequence of the previous lemma, observe the following.

(4f) Bounded Distortion Property $(B D P)$ : There exists $K \geq 1$ such that for all $\omega \in I_{A}^{*}$ and all $x, y \in X_{t o(\omega)}$

$$
\left\|\varphi_{\omega}^{\prime}(y)\right\| \leq K\left\|\varphi_{\omega}^{\prime}(x)\right\|
$$

In a different vein, it was proved in [4] that for each $t \geq 0$ the following limit exists (and can be equal to $\infty$ but not $-\infty)$ :

$$
\mathrm{P}_{\Phi}(t)=\lim _{n \rightarrow \infty} \frac{1}{n} \log \sum_{\omega \in I_{A}^{n}}\left\|\varphi_{\omega}^{\prime}\right\|^{t} .
$$

This number is called the topological pressure of the parameter $t$. It is important to notice that pressure is well defined whenever (4a) and (4c) are satisfied.

From the graph of the pressure function, two fundamental parameters were identified in [4]. First, Bowen's parameter $B P(\Phi)$ of a WPO CGDMS $\Phi$ is defined as

$$
B P(\Phi):=\inf \left\{t \geq 0: \mathrm{P}_{\Phi}(t)<0\right\} .
$$

A second useful parameter is the finiteness parameter

$$
\theta(\Phi)=\inf \left\{t \geq 0: \mathrm{P}_{\Phi}(t)<\infty\right\}=\sup \left\{t \geq 0: \mathrm{P}_{\Phi}(t)=\infty\right\} .
$$

It turns out that the pressure function is non-increasing on $[0, \infty)$, strictly decreasing on $[\theta(\Phi), \infty)$, and convex and continuous on $(\theta(\Phi), \infty)$. It is also right-continuous at $\theta(\Phi)$.

We now recall Mauldin and Urbanski's classification of systems in terms of the pressure function.

Definition 5.3. A WPO CGDMS $\Phi$ is said to be regular if there exists $t \geq 0$ such that $\mathrm{P}_{\Phi}(t)=0$. Otherwise, $\Phi$ is said to be irregular.

Definition 5.4. A WPO CGDMS is said to be strongly regular if there exists $t \geq 0$ such that $0<\mathrm{P}_{\Phi}(t)<\infty$.

Definition 5.5. A WPO CGDMS is said to be cofinitely regular if all of its cofinite subsystems are regular. (A family $\left.\Phi\right|_{F}:=\left\{\varphi_{i}\right\}_{i \in F}$ is said to be a cofinite subsystem of a system $\Phi=\left\{\varphi_{i}\right\}_{i \in I}$ if $F \subset I$ and the difference $I \backslash F$ is finite.)

The following fact, which relates the diverse kinds of regularity, can be found in [4]. 
Proposition 5.6. Each cofinitely regular system is strongly regular, and each strongly regular system is regular.

Finally, we state the central result of the theory of CGDMS. Let $\mathcal{F}$ in $(I)$ denote the family of all finite subsets of $I$. The following characterization of $\operatorname{HD}\left(J_{\Phi}\right)$, the Hausdorff dimension of the limit set $J_{\Phi}$, is a variant of Bowen's formula. It was proved in [4] as Theorem 4.2.13 for finitely primitive CGDMS, but the proof can easily be improved to finitely irreducible CGDMS.

Theorem 5.7. If a CGDMS $\Phi$ is finitely irreducible, that is, has an associated matrix $A$ which is finitely irreducible, then

$$
\operatorname{HD}\left(J_{\Phi}\right)=B P(\Phi)=\sup \left\{\operatorname{HD}\left(J_{\left.\Phi\right|_{F}}\right): F \in \mathcal{F} \text { in }(I)\right\} \geq \theta(\Phi) .
$$

It follows immediately from this theorem that if $\Phi$ is regular, that is if $\mathrm{P}_{\Phi}(t)=0$ for some $t$, then that $t$ is the only zero of the pressure function $\mathrm{P}_{\Phi}(t)$ and $t=\operatorname{HD}\left(J_{\Phi}\right)$. Moreover, a system $\Phi$ is strongly regular if and only if $\operatorname{HD}\left(J_{\Phi}\right)>\theta(\Phi)$.

Note also that the theorem is generally not true when there are overlaps, that is when the OSC is not satisfied.

\section{Analyticity of Perron-Frobenius operators}

Throughout this section $d \geq 3$. Fix a countable (finite or infinite) set $I$ and an incidence matrix $A: I \times I \rightarrow\{0,1\}$. Fix also $E \in M_{d}^{I} \subset\left(\mathbb{R}^{q}\right)^{I}$ such that $\Phi_{E}=\left(\varphi_{E_{i}}\right)_{i \in I}$ is a WPO CGDMS with incidence matrix $A_{E_{i_{1}} E_{i_{2}}}:=A_{i_{1} i_{2}}$, a finite vertex set $V_{E}$, and corresponding pairs $\left(X_{v}, W_{v}\right), v \in V_{E}$. Denote the common contraction rate by $s=s_{E} \in(0,1)$. Put

$$
R_{E}:=\inf \left\{R_{s}\left(\left(X_{t o\left(E_{i}\right)}, W_{t o\left(E_{i}\right)}\right) ;\left(X_{i n\left(E_{i}\right)}, W_{i n\left(E_{i}\right)}\right)\right): i \in I\right\}>0,
$$

where the number $R_{s}\left(\left(X_{t o\left(E_{i}\right)}, W_{t o\left(E_{i}\right)}\right) ;\left(X_{i n\left(E_{i}\right)}, W_{i n\left(E_{i}\right)}\right)\right)>0$ results from the application of Lemma 3.7 with $X=X_{t o\left(E_{i}\right)}, V=W_{t o\left(E_{i}\right)}$ and $s=s_{E}$. Note that the number $R_{E}$ is positive since it is the infimum of a finite set of positive numbers. Set also

$$
\Gamma_{E_{i}}:=B_{\mathbb{C}^{q}}\left(E_{i}, \delta_{E_{i}}\left(R_{E}\right)\right) \cap \mathbb{C} M_{d}
$$

for each $i \in I$, where each number $\delta_{E_{i}}\left(R_{E}\right)>0$ results from the application of Lemma 3.7 with $X=X_{t o\left(E_{i}\right)}, V=W_{t o\left(E_{i}\right)}, s=s_{E}$, and $e=E_{i}$. In view of Lemma 3.7, for each $D \in$ $\Pi_{i \in I} \Gamma_{E_{i}}$ the collection $\Phi_{D}=\left(\varphi_{D_{i}}\right)_{i \in I} \in\left(\mathbb{C} M_{d}\right)^{I} \subset\left(\mathbb{C}^{q}\right)^{I}$ forms a hyperbolic GDMS with contraction rate less than or equal to $(s+1) / 2$, the same set of vertices $V_{E}$, corresponding pairs $\left(\bar{B}_{\mathbb{C}^{d}}\left(X_{v}, R_{E}\right), B_{\mathbb{C}^{d}}\left(X_{v}, 2 R_{E}\right)\right), v \in V_{E}$, and the "same" incidence matrix generated by $A: I \times I \rightarrow\{0,1\}$, that is, $A_{D_{i_{1} D_{i_{2}}}}:=A_{i_{1} i_{2}}$.

However, the $\Phi_{D}$ 's are not WPO CGDMSs because they are not conformal in general. Nevertheless, since $\mathbb{C} M_{d}$ is open in $\mathbb{C}^{q}$, the set $\Pi_{i \in I} \Gamma_{E_{i}} \subset\left(\mathbb{C}^{q}\right)^{I}$ is open with respect to the box topology. Observe also that $\Pi_{i \in I}\left(\Gamma_{E_{i}} \cap M_{d}\right) \subset\left(\mathbb{R}^{q}\right)^{I}$ is open because $M_{d}$ is open in $\mathbb{R}^{q}$, and that if $D \in \Pi_{i \in I}\left(\Gamma_{E_{i}} \cap M_{d}\right)$, then $\Phi_{D}$ restricted to the generating pairs $\left(\bar{B}\left(X_{v}, R_{E}\right), B\left(X_{v}, 2 R_{E}\right)\right), v \in V_{E}$, is a WPO CGDMS. For every $D \in \Pi_{i \in I} \Gamma_{E_{i}}$, the coordinate projections $i \mapsto D_{i}$ induce the mapping $\omega \mapsto \omega_{D}=D_{\omega_{1}} D_{\omega_{2}} D_{\omega_{3}} \ldots \in\left\{D_{i}: i \in\right.$ $I\}_{A}^{\infty}$ defined on $I_{A}^{\infty}$. Note also that we will sometimes slightly abuse notation. For instance, we will shorten the notation for the coding maps from $\pi_{\Phi_{D}}$ to $\pi_{D}$. Our first result in this section is the following: 
Proposition 6.1. For every $\omega \in I_{A}^{\infty}$ the projection map $D \mapsto \pi_{D}\left(\omega_{D}\right) \in \mathbb{C}^{d}, D \in \Pi_{i \in I} \Gamma_{E_{i}}$, is holomorphic.

Proof. For every $v \in V_{E}$ fix a point $x_{v} \in X_{v}$. Then for every $D \in \prod_{i \in I} \Gamma_{E_{i}}$, we have

$$
\pi_{D}\left(\omega_{D}\right)=\lim _{n \rightarrow \infty} \varphi_{\left.\omega_{D}\right|_{n}}\left(x_{t o\left(\omega_{n}\right)}\right) \text {. }
$$

Now, let $U$ be the intersection of $\prod_{i \in I} \Gamma_{E_{i}}$ with some space box. Because of (3.6) and the first part of (3.1), for every $n \geq 1$ the mapping $D \mapsto \varphi_{\left.\omega_{D}\right|_{n}}\left(x_{t o\left(\omega_{n}\right)}\right), D \in U$, is holomorphic. Since the ranges of these maps are bounded (in fact, contained in $\cup_{v \in V_{E}} \bar{B}_{\mathbb{C}^{d}}\left(X_{v}, R_{E}\right)$ ), we conclude that the sequence of maps $D \mapsto \varphi_{\left.\omega_{D}\right|_{n}}\left(x_{t o\left(\omega_{n}\right)}\right), n \geq 1$, is normal. Consequently, all its limit points are holomorphic functions. Thus, $D \mapsto \pi_{D}\left(\omega_{D}\right)=\lim _{n \rightarrow \infty} \varphi_{\left.\omega_{D}\right|_{n}}\left(x_{t o\left(\omega_{n}\right)}\right)$ is holomorphic.

Furthermore, we have the following property.

Lemma 6.2. The family of projection maps $\left\{D \mapsto \pi_{D}\left(\omega_{D}\right)\right\}_{\omega \in I_{A}^{\infty}}$ is equicontinuous at the point $E$.

Proof. Fix $\varepsilon>0$, and for every $i \in I$ take $\xi_{i}>0$ so small that $B_{\mathbb{C}^{q}}\left(E_{i}, \xi_{i}\right) \subset \Gamma_{E_{i}}$ and that according to Lemma 3.5 (applied with $X=X_{t o\left(E_{i}\right)}, V=W_{t o\left(E_{i}\right)}$ and $e=E_{i}$ ) we have

$$
\sup \left\{\left\|\varphi_{f}-\varphi_{E_{i}}\right\|_{X_{t o\left(E_{i}\right)}}: f \in B_{\mathbb{C}^{q}}\left(E_{i}, \xi_{i}\right)\right\} \leq \varepsilon \text {. }
$$

For every $n \geq 1$, set

$$
\Delta_{n}:=\sup \left\{\left\|\varphi_{\left.\omega_{D}\right|_{n}}-\varphi_{\left.\omega_{E}\right|_{n}}\right\|_{X_{t o\left(\omega_{n}\right)}}: D \in \Pi_{i \in I} B_{\mathbb{C}^{q}}\left(E_{i}, \xi_{i}\right), \omega \in I_{A}^{\infty}\right\}
$$

and observe that $\Delta_{n} \leq \sqrt{d}\left(\max \left\{\operatorname{diam}\left(X_{v}\right): v \in V_{E}\right\}+2 R_{E}\right)$. For every $n \geq 1$, every $D \in \Pi_{i \in I} B_{\mathbb{C}^{q}}\left(E_{i}, \xi_{i}\right)$, every $\omega \in I_{A}^{\infty}$ and every $x \in X_{t o\left(\omega_{n+1}\right)}$, we have

$$
\begin{aligned}
\left\|\varphi_{\left.\omega_{D}\right|_{n+1}}(x)-\varphi_{\left.\omega_{E}\right|_{n+1}}(x)\right\| & =\left\|\begin{array}{r}
\left(\varphi_{\left.\omega_{D}\right|_{n}}\left(\varphi_{\left(\omega_{D}\right)_{n+1}}(x)\right)-\varphi_{\left.\omega_{D}\right|_{n}}\left(\varphi_{\left(\omega_{E}\right)_{n+1}}(x)\right)\right) \\
+\left(\varphi_{\left.\omega_{D}\right|_{n}}\left(\varphi_{\left(\omega_{E}\right)_{n+1}}(x)\right)-\varphi_{\left.\omega_{E}\right|_{n}}\left(\varphi_{\left(\omega_{E}\right)_{n+1}}(x)\right)\right)
\end{array}\right\| \\
& \leq\left(\frac{s+1}{2}\right)^{n}\left\|\varphi_{\left(\omega_{D}\right)_{n+1}}(x)-\varphi_{\left(\omega_{E}\right)_{n+1}}(x)\right\|+\Delta_{n} \\
& \leq\left(\frac{s+1}{2}\right)^{n}\left\|\varphi_{\left(\omega_{D}\right)_{n+1}}-\varphi_{\left(\omega_{E}\right)_{n+1}}\right\|_{X_{t o\left(\omega_{n+1}\right)}}+\Delta_{n} \\
& \leq\left(\frac{s+1}{2}\right)^{n} \varepsilon+\Delta_{n} .
\end{aligned}
$$

Therefore

Thus, by induction,

$$
\Delta_{n+1} \leq \Delta_{n}+\varepsilon\left(\frac{s+1}{2}\right)^{n}
$$

$$
\Delta_{n} \leq \varepsilon \sum_{j=1}^{n}\left(\frac{s+1}{2}\right)^{j} \leq \varepsilon \sum_{j=0}^{\infty}\left(\frac{s+1}{2}\right)^{j}=\frac{\varepsilon}{1-\frac{s+1}{2}}=\frac{2 \varepsilon}{1-s} .
$$

Applying (6.1), we obtain that $\left\|\pi_{D}\left(\omega_{D}\right)-\pi_{E}\left(\omega_{E}\right)\right\| \leq 2 \varepsilon /(1-s)$. Since $\varepsilon>0$ was chosen arbitrarily, the family $\left\{D \mapsto \pi_{D}\left(\omega_{D}\right)\right\}_{\omega \in I_{A}^{\infty}}$ is equicontinuous at $E$. 
Now, for every $\omega \in I_{A}^{\infty}$ define the function $\psi_{\omega}: \Pi_{i \in I} \Gamma_{E_{i}} \rightarrow \mathbb{C}$ by

$$
\psi_{\omega}(D):=\frac{\left\|\varphi_{\left(\omega_{D}\right)_{1}}^{\prime}\left(\pi_{D}\left(\sigma \omega_{D}\right)\right)\right\| \|}{\left\|\varphi_{\left(\omega_{E}\right)_{1}}^{\prime}\left(\pi_{E}\left(\sigma \omega_{E}\right)\right)\right\|} .
$$

where $\left\|\left|\varphi_{f}^{\prime}(z) \|\right| \mid\right.$ was defined in (3.7), while $\| \varphi_{e}^{\prime}(x) \|$ is the scaling factor of $\varphi_{e}$ given in (3.4). Note that it follows immediately from Proposition 6.1 that each function $\psi_{\omega}, \omega \in I_{A}^{\infty}$, is holomorphic. Moreover, we have the following.

Lemma 6.3. For every $i \in I$ there exists $\rho_{i}>0$ such that $B_{\mathbb{C}^{q}}\left(E_{i}, \rho_{i}\right) \subset \Gamma_{E_{i}}$ and

$$
\left|\psi_{\omega}(D)-1\right|<\frac{1}{2}
$$

for all $\omega \in I_{A}^{\infty}$ and all $D \in \Pi_{i \in I} B_{\mathbb{C}^{q}}\left(E_{i}, \rho_{i}\right)$.

Proof. This lemma is a direct consequence of (3.4), (3.7), Lemma 3.3 and Lemma 6.2.

Now, let $\log : B_{\mathbb{C}}(1,1 / 2) \rightarrow \mathbb{C}$ be the branch of the logarithm determined by the requirement that $\log 1=0$. With $\rho_{i}$ as specified above, for every $D \in \prod_{i \in I} B_{\mathbb{C}^{q}}\left(E_{i}, \rho_{i}\right)$ and every $\omega \in I_{A}^{\infty}$, set

$$
\zeta_{\omega}(D):=\log \psi_{\omega}(D)+\log \left\|\varphi_{\left(\omega_{E}\right)_{1}}^{\prime}\left(\pi_{E}\left(\sigma \omega_{E}\right)\right)\right\| .
$$

Fix $t_{0}>\theta\left(\Phi_{E}\right)$, where $\theta\left(\Phi_{E}\right)$ is the finiteness parameter of the WPO CGDMS $\Phi_{E}$. Aiming to apply Theorem 4.5, we shall now prove the main result of this section:

Lemma 6.4. For every $(t, D) \in\left\{\xi \in \mathbb{C}: \operatorname{Re}(\xi)>t_{0}\right\} \times \Pi_{i \in I} B_{\mathbb{C}^{q}}\left(E_{i}, \rho_{i}\right)$ we have that $t \zeta_{(\cdot)}(D) \in \mathcal{K}_{\frac{s+1}{2}}$. Moreover, the function $(t, D) \mapsto \mathcal{L}_{t \zeta_{(\cdot)}(D)} \in L\left(\mathcal{H}_{\frac{s+1}{2}}^{b}\right),(t, D) \in\{\xi \in \mathbb{C}:$ $\left.\operatorname{Re}(\xi)>t_{0}\right\} \times \Pi_{i \in I} B_{\mathbb{C}^{q}}\left(E_{i}, \rho_{i} / 2\right)$ is holomorphic.

Proof. Fix $(t, D) \in\left\{\xi \in \mathbb{C}: \operatorname{Re}(\xi)>t_{0}\right\} \times \Pi_{i \in I} B_{\mathbb{C}^{q}}\left(E_{i}, \rho_{i}\right)$. Since, by Lemma 6.3, $\left|\log \psi_{(\cdot)}(D)\right|$ is uniformly bounded by $\log (3 / 2)$, summability of the potential $t \zeta_{(\cdot)}(D)$ follows immediately from the choice of $t_{0}$.

We shall now check that the function $t \zeta_{(\cdot)}(D) \in \mathcal{H}_{\frac{s+1}{2}}$. In view of $(3.7)$, for all $\omega, \tau \in I_{A}^{\infty}$ with $\omega_{1}=\tau_{1}$, we have

$$
\left|t \zeta_{\tau}(D)-t \zeta_{\omega}(D)\right|=|t|\left|\log \left(\frac{\|\left.\left|\pi_{D}\left(\sigma \omega_{D}\right)-a_{D}\right|\right|^{2}}{\|\left.\left|\pi_{D}\left(\sigma \tau_{D}\right)-a_{D}\right|\right|^{2}}\right)\right|,
$$

where $a_{D}:=a_{\left(\omega_{D}\right)_{1}}=a_{\left(\tau_{D}\right)_{1}}$. Thanks to our choice of the $\Gamma_{E_{i}}$ 's and because $B_{\mathbb{C}^{q}}\left(E_{i}, \rho_{i}\right) \subset$ $\Gamma_{E_{i}}$ for each $i \in I$, we can apply Lemma 3.3 with $X=X_{t o\left(E_{\omega_{1}}\right)} \ni \pi_{E}\left(\sigma \omega_{E}\right), V=W_{t o\left(E_{\omega_{1}}\right)}$, $z=\pi_{D}\left(\sigma \omega_{D}\right), w=\pi_{D}\left(\sigma \tau_{D}\right)$, and $\alpha=\beta=a_{D}$. With $C_{1}:=\max _{v \in V_{E}} C\left(X_{v}, W_{v}\right), C_{2}:=$ $\max \left\{\operatorname{diam}\left(X_{v}\right): v \in V_{E}\right\}+2 R_{E}$ and $C_{3}:=\max \left\{\operatorname{diam}\left(X_{v}\right): v \in V_{E}\right\}$, it follows that

$$
\begin{aligned}
\left|\frac{\|\| \pi_{D}\left(\sigma \omega_{D}\right)-a_{D}||^{2}}{\|\| \pi_{D}\left(\sigma \tau_{D}\right)-a_{D}||^{2}}-1\right| & \leq C_{1} \max \left\{\left\|\pi_{D}\left(\sigma \omega_{D}\right)-\pi_{D}\left(\sigma \tau_{D}\right)\right\|^{2},\left\|\pi_{D}\left(\sigma \omega_{D}\right)-\pi_{D}\left(\sigma \tau_{D}\right)\right\|\right\} \\
& \leq C_{1} C_{2}\left\|\pi_{D}\left(\sigma \omega_{D}\right)-\pi_{D}\left(\sigma \tau_{D}\right)\right\| \\
& \leq C_{1} C_{2} C_{3}\left(\frac{s+1}{2}\right)^{|\omega \wedge \tau|-1} .
\end{aligned}
$$


Thus, there exists a constant $C_{4} \geq 0$ such that

$$
\left|t \zeta_{\tau}(D)-t \zeta_{\omega}(D)\right| \leq C_{4}|t|\left(\frac{s+1}{2}\right)^{|\omega \wedge \tau|} .
$$

So $t \zeta_{(\cdot)}(D) \in \mathcal{H}_{\frac{s+1}{2}}$, and in consequence $t \zeta_{(\cdot)}(D) \in \mathcal{K}_{\frac{s+1}{2}}$. On the other hand, it follows immediately from Proposition 6.1 that for every $\omega \in I_{A}^{\infty}$, the function $D \mapsto \psi_{\omega}(D)$ is holomorphic, and consequently $(t, D) \mapsto t \zeta_{\omega}(D) \in \mathbb{C},(t, D) \in\left\{\xi \in \mathbb{C}: \operatorname{Re}(\xi)>t_{0}\right\} \times$ $\Pi_{i \in I} B_{\mathbb{C}^{q}}\left(E_{i}, \rho_{i}\right)$, is holomorphic. In view of Theorem 4.5 and Hartogs Theorem, in order to complete the proof, it suffices to demonstrate that the function $(t, D) \mapsto t \zeta_{(\cdot)}(D) \in$ $\mathcal{K}_{\frac{s+1}{2}}$, with $(t, D)$ in a one-dimensional space box neighbourhood of $\left(t_{0}, E\right)$, is continuous. Obviously, this function is continuous with respect to the variable $t$. So, we may assume that $t$ is fixed and $D$ is a one-dimensional space box neighbourhood of $\prod_{i \in I} B_{\mathbb{C}^{q}}\left(E_{i}, \rho_{i}\right)$, say $D \in B_{\mathbb{C}}\left(b, \rho_{i}\right)$ for some fixed $i \in I$. Fix $\omega \in I_{A}^{\infty}$. Since the function $D \mapsto \log \psi_{\omega}(D)$ is holomorphic and bounded in modulus by $\log (3 / 2)$ on $B_{\mathbb{C}}\left(b, \rho_{i}\right)$, and satisfies $\log \psi_{\omega}(E)=0$, its Taylor series expansion on $B_{\mathbb{C}}\left(b, \rho_{i}\right)$ takes on the form

$$
\log \psi_{\omega}(D)=\sum_{n=1}^{\infty} a_{n}(\omega)(D-b)^{n}
$$

and from Cauchy's estimates

$$
\left|a_{n}(\omega)\right| \leq \rho_{i}^{-n} \log (3 / 2) .
$$

Hence if $F \in B_{\mathbb{C}}\left(b, \rho_{i}\right)$, then

$$
\begin{aligned}
\log \psi_{\omega}(F)-\log \psi_{\omega}(D) & =\sum_{n=1}^{\infty} a_{n}(\omega)\left((F-b)^{n}-(D-b)^{n}\right) \\
& =\sum_{n=1}^{\infty} a_{n}(\omega)(F-D) \sum_{j=0}^{n-1}(F-b)^{n-1-j}(D-b)^{j}
\end{aligned}
$$

Consequently, if $D, F \in B_{\mathbb{C}}\left(b, \rho_{i} / 2\right)$, then

$$
\begin{aligned}
\left|\log \psi_{\omega}(F)-\log \psi_{\omega}(D)\right| & \leq \sum_{n=1}^{\infty} \rho_{i}^{-n} \log (3 / 2)|F-D| \sum_{j=0}^{n-1}|F-b|^{n-1-j}|D-b|^{j} \\
& \leq \log (3 / 2)|F-D| \sum_{n=1}^{\infty} \rho_{i}^{-n} \sum_{j=0}^{n-1}\left(\rho_{i} / 2\right)^{n-1} \\
& =\log (3 / 2) \rho_{i}^{-1}\left(\sum_{n=1}^{\infty} n 2^{-(n-1)}\right)|F-D| .
\end{aligned}
$$

It then follows from (6.3) that

$$
\left\|t \zeta_{(\cdot)}(F)-t \zeta_{(\cdot)}(D)\right\|_{\infty} \leq \log (3 / 2)|t| \rho_{i}^{-1}\left(\sum_{n=1}^{\infty} n 2^{-(n-1)}\right)|F-D| .
$$


Now, fix $\omega, \tau \in I_{A}^{\infty}$ with $\omega_{1}=\tau_{1}$. It follows from (6.3) and (6.4) that

$$
\left|\log \psi_{\tau}(D)-\log \psi_{\omega}(D)\right| \leq C_{4}\left(\frac{s+1}{2}\right)^{|\omega \wedge \tau|} .
$$

Combining this with (6.5), Cauchy's estimates yield

$$
\left|a_{n}(\tau)-a_{n}(\omega)\right| \leq C_{4} \rho_{i}^{-n}\left(\frac{s+1}{2}\right)^{|\omega \wedge \tau|} .
$$

So, if $D, F \in B_{\mathbb{C}}\left(b, \rho_{i} / 2\right)$, we get using (6.6) that

$$
\begin{aligned}
\mid\left(\log \psi_{\tau}(F)-\right. & \left.\log \psi_{\tau}(D)\right)-\left(\log \psi_{\omega}(F)-\log \psi_{\omega}(D)\right) \mid \\
& =\left|\sum_{n=1}^{\infty}\left(a_{n}(\tau)-a_{n}(\omega)\right)(F-D) \sum_{j=0}^{n-1}(F-b)^{n-1-j}(D-b)^{j}\right| \\
& \leq C_{4} \rho_{i}^{-1}\left(\sum_{n=1}^{\infty} n 2^{-(n-1)}\right)|F-D|\left(\frac{s+1}{2}\right)^{|\omega \wedge \tau|} .
\end{aligned}
$$

Now, given a function $g: I_{A}^{\infty} \rightarrow \mathbb{C}$ we denote (as the reader will recall) by $v_{s}(g)$ the $s$ variation of $g$, that is, $v_{s}(g):=\sup \left\{|g(\omega)-g(\tau)| / s^{n}:\left.\omega\right|_{n}=\left.\tau\right|_{n}\right\}$, and by $\|g\|_{s}$ the $s$-norm of $g$, namely $\|g\|_{s}:=\|g\|_{\infty}+v_{s}(g)$ (cf. section 4). Then it follows immediately from (6.8) that

$$
v_{\frac{s+1}{2}}\left(\log \psi_{(\cdot)}(F)-\log \psi_{(\cdot)}(D)\right) \leq C_{4} \rho_{i}^{-1}\left(\sum_{n=1}^{\infty} n 2^{-(n-1)}\right)|F-D|,
$$

and consequently

$$
v_{\frac{s+1}{2}}\left(t \zeta_{(\cdot)}(F)-t \zeta_{(\cdot)}(D)\right) \leq C_{4}|t| \rho_{i}^{-1}\left(\sum_{n=1}^{\infty} n 2^{-(n-1)}\right)|F-D| .
$$

Together with (6.7), this yields

$$
\left\|t \zeta_{(\cdot)}(F)-t \zeta_{(\cdot)}(D)\right\|_{\frac{s+1}{2}} \leq\left(C_{4}+\log (3 / 2)\right)|t| \rho_{i}^{-1}\left(\sum_{n=1}^{\infty} n 2^{-(n-1)}\right)|F-D| .
$$

Thus the proof of continuity is complete and we are done.

\section{Real analyticity of Hausdorff dimension}

Sticking to the same framework as in the preceding section, $d \geq 3, I$ is a countable (finite or infinite) alphabet and $A: I \times I \rightarrow\{0,1\}$ an incidence matrix. Fix $E \in M_{d}^{I} \subset$ $\left(\mathbb{R}^{q}\right)^{I}$ such that $\Phi_{E}=\left(\varphi_{E_{i}}\right)_{i \in I}$ is a strongly regular WPO CGDMS with a finite set of vertices $V_{E}$ and corresponding pairs $\left(X_{v}, W_{v}\right), v \in V_{E}$. To each element $D \in \Pi_{i \in I} \Gamma_{E_{i}}$, ascribe uniquely a hyperbolic GDMS $\Phi_{D}=\left(\varphi_{D_{i}}\right)_{i \in I} \in \mathbb{C} M_{d}^{I} \subset\left(\mathbb{C}^{q}\right)^{I}$ as in the previous section. Recall that if $D \in \Pi_{i \in I}\left(\Gamma_{E_{i}} \cap M_{d}\right)$, then $\Phi_{D}$ restricted to the generating pairs $\left(\bar{B}\left(X_{v}, R_{E}\right), B\left(X_{v}, 2 R_{E}\right)\right)_{v \in V_{E}}$ is a WPO CGDMS. Moreover, the limit sets of the restricted and unrestricted systems $\Phi_{D}$ are the same and contained in $\mathbb{R}^{d}$. The first result of this section is the following. 
Theorem 7.1. For every $i \in I$ there is $R_{i}>0$ such that the function $D \mapsto B P\left(\Phi_{D}\right)$, $D \in \Pi_{i \in I} B\left(E_{i}, R_{i}\right)$, is real-analytic.

Proof. For every $t \in \mathbb{C}$ with $\operatorname{Re}(t)>\theta\left(\Phi_{E}\right)$ and $D \in \Pi_{i \in I} \Gamma_{E_{i}}$ put $\mathcal{L}_{t, D}:=\mathcal{L}_{t \zeta_{(\cdot)}(D)} \in$ $L\left(\mathcal{H}_{\frac{s+1}{2}}^{b}\right)$. Fix a space box $U \subset \mathbb{C} \times \prod_{i \in I} B_{\mathbb{C}^{q}}\left(E_{i}, \rho_{i} / 2\right)$ containing $\left(t_{0}, E\right)$ (with $\rho_{i}$ is as defined in Lemma 6.3). Apply Lemma 6.4 with $t_{0}=t_{E}:=B P\left(\Phi_{E}\right)>\theta\left(\Phi_{E}\right)$ (the strict inequality holds since $\Phi_{E}$ is strongly regular). In view of that lemma and Theorem 4.4, Kato-Rellich perturbation theorem applies and yields $R_{i} \in\left(0, \rho_{i} / 2\right.$ ] (in fact, $R_{i}=\rho_{i} / 2$ except for finitely many $i \in I)$ and a holomorphic function $\lambda: U \cap\left(\mathbb{C} \times \prod_{i \in I} B_{\mathbb{C}^{q}}\left(E_{i}, R_{i}\right)\right) \rightarrow \mathbb{C}$ such that $\lambda\left(t_{E}, E\right)=e^{P_{E}\left(t_{E}\right)}$ and such that $\lambda(t, D)$ is a simple isolated eigenvalue of the operator $\mathcal{L}_{t, D}: \mathcal{H}_{\frac{s+1}{2}}^{b} \rightarrow \mathcal{H}_{\frac{s+1}{2}}^{b}$ with the remainder of the spectrum separated uniformly from $\lambda(t, D)$ In particular, decreasing $R_{i}>0$ appropriately, there is $\eta>0$ such that

$$
\sigma\left(\mathcal{L}_{t, D}\right) \cap B_{\mathbb{C}}\left(e^{P_{E}\left(t_{E}\right)}, \eta\right)=\{\lambda(t, D)\}
$$

for all $(t, D) \in U$, where $\sigma\left(\mathcal{L}_{t, D}\right)$ denotes the spectrum of $\mathcal{L}_{t, D}$. Since $e^{P_{D}(t)}$ is the spectral radius $r\left(\mathcal{L}_{t, D}\right)$ of the operator $\mathcal{L}_{t, D}$ for all $(t, D) \in U \cap\left(\mathbb{R} \times M_{d}^{I}\right)$, in view of the semicontinuity of the spectral set function (see Theorem 10.20 on page 256 in [8]), by taking $R_{i}$ 's sufficiently small we also have that $r\left(\mathcal{L}_{t, D}\right) \in\left[0, e^{P_{E}\left(t_{E}\right)}+\eta\right)$, and along with (7.1) this implies that $\lambda(t, D)=e^{P_{D}(t)}$ for all $(t, D) \in U \cap\left(\mathbb{R} \times M_{d}^{I}\right)$. Consequently, the function $(t, D) \mapsto P_{D}(t)$, $(t, D) \in U \cap\left(\mathbb{R} \times M_{d}^{I}\right)$, is real-analytic. Since $P_{E}\left(t_{E}\right)=0$ and since

$$
\left.\frac{\partial P}{\partial t}\right|_{\left(t_{E}, E\right)}=\int \log \left\|\varphi_{\left(\omega_{E}\right)_{1}}^{\prime}\left(\pi_{E}\left(\sigma \omega_{E}\right)\right)\right\| d \mu_{E}(\omega)<0,
$$

where $\mu_{E}$ is the Gibbs (equilibrium) state of the potential $\omega \mapsto t_{E} \log \left\|\varphi_{\left(\omega_{E}\right) 1}^{\prime}\left(\pi_{E}\left(\sigma \omega_{E}\right)\right)\right\|$, it follows from the implicit function theorem that, with appropriately smaller $R_{i}$ 's, there exists a real-analytic function $t: \hat{U} \rightarrow \mathbb{R}$ defined on $\hat{U}$, the projection of $U$ onto the set $\Pi_{i \in I} B\left(E_{i}, R_{i}\right)$, such that $t(E)=t_{E}$ and $P_{D}(t(D))=0$. Hence $t(D)=B P\left(\Phi_{D}\right)$ and we are done.

As an immediate consequence of this theorem and Bowen's formula, we obtain the following:

Theorem 7.2. Suppose that the incidence matrix $A: I \times I \rightarrow\{0,1\}$ is finitely irreducible and that $\Phi_{E}$ is a strongly regular CGDMS. If with $R_{i}, i \in I$, as in Theorem 7.1 or smaller, all the systems $\Phi_{D}, D \in \Pi_{i \in I} B\left(E_{i}, R_{i}\right)$, satisfy the $O S C$, then the Hausdorff dimension function $D \mapsto \operatorname{HD}\left(J_{\Phi_{D}}\right), D \in \prod_{i \in I} B\left(E_{i}, R_{i}\right)$, is real-analytic.

Remark 7.3. Suppose that the incidence matrix $A: I \times I \rightarrow\{0,1\}$ is finitely irreducible and that $\Phi_{E}=\left(\varphi_{E_{i}}\right)_{i \in I}$ is a strongly regular CGDMS. If $\Phi_{E}$ satisfies the super strong separation condition, that is, if for every $i \in I$

$$
\varphi_{E_{i}}\left(X_{t o\left(E_{i}\right)}\right) \bigcap \overline{\bigcup_{j \in I \backslash\{i\}} \varphi_{E_{j}}\left(X_{t o\left(E_{j}\right)}\right)}=\emptyset,
$$

then by proceeding inductively with respect to a good order on $I$, we can construct $R_{i}^{*} \in$ $(0, \infty)$ such that for each $D \in \Pi_{i \in I} B\left(E_{i}, R_{i}^{*}\right)$, the system $\Phi_{D}$ also satisfies the super strong 
separation condition. In particular, each such $\Phi_{D}$ satisfies the OSC and Theorem 7.2 applies to that family of systems.

\section{REFERENCES}

[1] L. Baribeau, M. Roy, Analytic multifunctions, holomorphic motions and Hausdorff dimension in IFSs, Monatshefte für Mathematik 147 (3) (2006), 199-217.

[2] R. Benedetti, C. Petronio, Lectures on Hyperbolic Geometry, Universitext, Springer-Verlag, Berlin, 1992.

[3] M. Urbański, Analytic Families of Semihyperbolic Generalized Polynomial-Like Mappings, Preprint 2006.

[4] R. D. Mauldin, M. Urbański, Graph Directed Markov Systems: Geometry and Dynamics of Limit Sets, Cambridge Tracts in Mathematics 148, Cambridge Univ. Press, 2003.

[5] R. D. Mauldin, M. Urbański, Gibbs states on the symbolic space over an infinite alphabet, Israel J. of Math. 125 (2001), 93-130.

[6] R. D. Mauldin, M. Urbański, Dimensions and measures in infinite iterated function systems, Proc. London Math. Soc. (3) 73 (1996), 105-154.

[7] M. Roy, M. Urbanski, Regularity properties of Hausdorff dimension in infinite conformal IFSs, Erg. Th. Dyn. Sys. 25 (6) (2005), 1961-1983.

[8] W. Rudin, Functional Analysis, Second edition, International Series in Pure and Applied Mathematics, McGraw-Hill, New York, 1991.

[9] O. Sarig, Thermodynamic formalism for countable Markov shifts, Erg. Th. Dyn. Sys. 19 (1999), 15651593.

[10] J. Väisälä, Lectures on n-Dimensional Quasiconformal Mappings, Lect. Notes in Math. 229, SpringerVerlag, Berlin — New York, 1971.

Mario Roy, Glendon College, York University, 2275 Bayview Avenue, Toronto, Canada, M4N 3M6

E-mail address: mroy@gl.yorku.ca

Mariusz Urbański, Department of Mathematics, University of North Texas, Denton, TX 76203-1430, USA

E-mail address: urbanski@unt.edu

Web: www.math.unt.edu/ urbanski 